Zhang Yongyong (Orcid ID: 0000-0001-9159-9626)

Pang Xuan (Orcid ID: 0000-0003-4041-0007)

Shao Quanxi (Orcid ID: 0000-0002-9768-137X)

She Dunxian (Orcid ID: 0000-0003-4660-3301)

Sun Jianqi (Orcid ID: 0000-0002-3879-6986)

Confidential manuscript submitted to Journal of Geophysical Research: Atmosphere

\title{
Regional patterns of extreme precipitation and urban signatures in metropolitan areas
}

\section{Yongyong Zhang ${ }^{1 \dagger}$, Xuan Pang ${ }^{2}$, Jun Xia ${ }^{3}$, Quanxi Shao ${ }^{4}$, Entao Yu ${ }^{5}$, Tongtiegang Zhao ${ }^{6}$, Dunxian She $^{7}$, Jianqi Sun ${ }^{8}$, Jingjie Y ${ }^{9}$, Xinyao Pan ${ }^{10}$, Xiaoyan Zhai ${ }^{11}$}

${ }^{1}$ Key Laboratory of Water Cycle and Related Land Surface Processes, Institute of Geographic Sciences and Natural Resources Research, Chinese Academy of Sciences, Beijing, 100101, China.

${ }^{2}$ Key Laboratory of Water Cycle and Related Land Surface Processes, Institute of Geographic Sciences and Natural Resources Research, Chinese Academy of Sciences, Beijing, 100101, China.

${ }^{3}$ State Key Laboratory of Water Resources \& Hydropower Engineering Sciences, Wuhan University, Wuhan 430072, China.

${ }^{4}$ CSIRO Data 61, Private Bag 5, Wembley, Western Australia 6913, Australia.

${ }^{5}$ Nansen-Zhu International Research Centre, Institute of Atmospheric Physics, Chinese Academy of Sciences, Beijing 100029, China; Collaborative Innovation Center on Forecast and Evaluation of Meteorological Disasters, Nanjing University of Information Science and Technology, Nanjing 210044, China

${ }^{6}$ Department of Water Resources and Environment, Sun Yat-Sen University.

${ }^{7}$ State Key Laboratory of Water Resources \& Hydropower Engineering Sciences, Wuhan University, Wuhan 430072, China.

${ }^{8}$ Nansen-Zhu International Research Centre, Institute of Atmospheric Physics, Chinese Academy of Sciences, Beijing 100029, China; Collaborative Innovation Center on Forecast and Evaluation of Meteorological Disasters, Nanjing University of Information Science and Technology, Nanjing 210044, China

${ }^{9}$ Key Laboratory of Water Cycle and Related Land Surface Processes, Institute of Geographic Sciences and Natural Resources Research, Chinese Academy of Sciences, Beijing, 100101, China.

${ }^{10}$ Beijing Water Science and Technology Institute, Beijing 100048, China..

${ }^{11}$ China Institute of Water Resources and Hydropower Research, Beijing 100038, China.

Corresponding author: Yongyong Zhang (zhangyy003@igsnrr.ac.cn)

This is the author manuscript accepted for publication and has undergone full peer review but has not been through the copyediting, typesetting, pagination and proofreading process, which may lead to differences between this version and the Version of Record. Please cite this article as doi: 10.1029/2018JD029718

This article is protected by copyright. All rights reserved. 


\section{Key Points:}

- Extreme precipitation metrics in central urban region and windward slope of topographic area are greater than those in mountainous region

- Magnitude and frequency of extreme precipitation are enhanced by expansions of urban area, irregularity, patch, dominance and heat islands

- Duration of extreme precipitation is positive for the urban area and dominance, but negative for irregularity, patch and heat islands 


\begin{abstract}
Observations show that metropolitan areas throughout China, which are experiencing rapid urbanization, may have enhanced extreme precipitation. However, the underlying urban-induced mechanism is poorly understood, particularly for entire characteristics of extreme precipitation. Focusing on the Beijing metropolitan area, we investigate regional patterns of extreme precipitation characterized by magnitude, frequency, duration and timing metrics according to daily observations from 1975 to 2015 at 20 weather stations. Urbanization effects are explored by physical metrics of urbanization, including area, complexity, fragmentation and dominance deduced from five periods of land use maps. Results show that the magnitudes and frequencies of extreme precipitation have decreased over time, the consecutive precipitation days have been extended, and the Julian date of maximum precipitation (DatPmx) has been delayed. Temporal trends at $\sim 40 \%$ of weather stations are significant. According to precipitation metrics and geographical features, three representative regions are identified: the central urban region, the windward slope of topographic area and the mountainous region. Compared with the metrics in mountainous region, the magnitudes of windward slope and central urban region are $24.3 \% \sim 60.6 \%$ and 5.9\% 47.3\% greater, respectively; the frequencies are increased by 1.17 and 1.10 days, respectively; and the average DatPmx values are delayed by 7.0 and 4.0 days, respectively. The magnitudes and frequencies are enhanced by expansion of urban area, complexity, fragmentation, dominance and heat islands. The durations are positive for urban area and dominance, but negative for urban complexity, fragmentation and heat islands. Furthermore, the effects in central urban region are more significant due to high urbanization rates.
\end{abstract}

Keywords: Extreme precipitation metrics; Urban signatures; Regional classification; Causal relationship; Metropolitan area

\title{
1 Introduction
}

Urbanization is inevitable due to global social and economic development. By the end of $2015,54.9 \%$ of the world's population lived in metropolitan areas, and this figure is projected to be $56.2 \%$ in developing countries and $81.5 \%$ in developed countries by 2030 (United Nations, Department of Economic and Social Affairs, Population Division, 2015). China is the largest developing country and has been at the rapid urbanization stage since the implementation of the reform and opening-up policy in 1978 (Xia et al., 2017). Currently, approximately 56.1\% of the Chinese population lives in metropolitan areas and this figure is projected to exceed $70 \%$ by 2030.

Metropolitan areas are experiencing the highest intensity of human activity, which leads to severe urban issues, such as water and air pollution, waterlogging, resource shortages, high housing prices and traffic jams (Xia et al., 2017). From a hydrometeorological perspective, 
urbanization, which involves population aggregation and impervious surface expansion, artificially modifies land use or cover and thus interferes with exchanges of heat, water and aerosols between the land surface and the atmosphere (Crutzen, 2004; Kaufmann et al., 2007). The consequences are a decrease in albedo and soil moisture, an increase in surface flow and air temperature differences between urban and surrounding rural regions (Bounoua et al., 2015; Karl and Knight, 1998; Song et al., 2014). Therefore, local micrometeorology (e.g., air temperature and precipitation) is manifested in metropolitan areas, whose characteristics are quite distinct from the climate patterns of rural surroundings (Oke, 1973; Shepherd, 2005; Zhao et al., 2014).

Extreme precipitation is a critical element of urban micrometeorology that coincides with urbanization (Shepherd, 2005; Kaufmann et al., 2007). Spatial and temporal variations of urban extreme precipitation have become a growing concern over decades (Lowry, 1998; Kaufmann et al., 2007; Song et al., 2014; Yang et al., 2014). However, most studies are limited to specific metrics related to precipitation magnitude, such as hourly precipitation magnitude (Jauregui and Romales,1996), total summer precipitation, heavy and moderate precipitation intensities (Yang et al., 2012), warm season precipitation and maximum hourly precipitation (Song et al., 2014), and ignore other attributes of extreme precipitation (e.g., frequency, duration and timing) which are also very helpful for the adaptation management of disaster weather. The existing studies are still deficient in capturing the entirety of extreme precipitation and its regional patterns. Furthermore, the urbanization-induced mechanism of extreme precipitation is still poorly understood (Kaufmann et al., 2007; Song et al., 2014; Yang et al., 2014; Zhao et al.,2018). The possible causes include convergence turbulence enhancement due to increased surface roughness and urban heat islands (UHIs) (Jauregui and Romales, 1996; Thielen et al., 2000), urban atmosphere humidification by moisture emission and transportation (Molders and Olson, 2004), and aerosol release for cloud condensation nuclei sources (Kaufmann et al., 2007). Most interpretations are probably deduced according to the precipitation differences between urban and rural surroundings based on surface station records, satellites or numerical models (Jauregui and Romales, 1996; Kalnay and Cai, 2003; Molders and Olson, 2004; Ramamurthy and BouZeid, 2014; Song et al., 2014). Kaufmann et al. (2007) used Granger causality to investigate the relationship between urban precipitation deficit and surface temperature, urbanization complexity during the dry season, and found that urbanization probably reduced local precipitation by changing surface properties and evapotranspiration. However, this complexity is only one physical characteristic of urbanization and extreme precipitation is not considered. Therefore, the causal relationship between extreme precipitation and other metrics of urban expansion is still less understood. Molders and Olson (2004) adopted NCAR Mesoscale Model (MM5) to examine the impact of urbanization on precipitation for Fairbanks, Alaska, but controlled experiments were only carried out by changing model input variables individually, e.g., urban area growth, release of aerosols, moisture and heat. Difficulties still exist, such as the determination of reliable emission scenarios, and accurate descriptions of physical processes (Molders and Olson, 2004; Ramamurthy and Bou-Zeid, 2014; Zhang et al., 2014). 
This paper aims to address three key questions regarding urbanization effects on extreme precipitation. The first is how to describe the entire characteristics of extreme precipitation and urbanization processes in a comprehensive manner, rather than only using precipitation intensity (magnitude) or urban area expansion? Second, do all the extreme precipitation metrics exist in regional discrepancies in the metropolitan region, particularly between metropolitan areas and rural surroundings? Third, what is the causal mechanism between extreme precipitation variations and urban expansion by Granger causality detection and a modelling approach from a regional climate model? To this end, we provide a comprehensive investigation to explore these three questions in the Beijing metropolitan region. Multiple physical metrics of urbanization are involved, including area, complexity, fragmentation and dominance, and extreme precipitation is entirely characterized by magnitude, frequency, duration and timing metrics. Long-term series of local climate observations from weather station networks, and land use maps with high resolution are adopted. This study is expected to provide insights into the extreme precipitation induced by urbanization and to support scientific urban landscape planning for the adaptation to urban precipitation islands.

\section{Materials and Methods}

\subsection{Study area}

Beijing is the capital city of China and the national center of politics, culture, international communication and scientific innovation. The Beijing municipality currently comprises 16 county-level administrative subdivisions, i.e., Dongcheng and Xicheng in the inner city, Haidian, Shijingshan, Fengtai and Chaoyang in the urban area; Changping, Shunyi, Tongzhou, Daxing, Fangshan and Mentougou in the inner suburb; and Yanqing, Huairou, Miyun and Pinggu in the outer suburb (Figure 1). Beijing is the third most populous city in the world and the second largest city in China with a population size of 21.7 million at the end of 2015. The city has experienced obvious urbanization over the past four decades and reached the standard of moderately developed countries by 2000. During the period of 2000-2017, the urbanization rate increased from $74.6 \%$ to $86.5 \%$, and the built-up area increased from $488 \mathrm{~km}^{2}$ to $1368 \mathrm{~km}^{2}$ (BMBS and SONBSB, 2015) .

The city is situated at the northern tip of the North China Plain, which opens to the south and east of the city, while mountains to the north, northwest and west shield the city. Beijing belongs to a cold climate region with a dry winter and hot summer. The annual average precipitation is approximately $570 \mathrm{~mm}$, of which three quarters fall in the summer (from June to August) (BWA, 2015). Therefore, extreme precipitation can occur easily, causing severe waterlogging and inducing traffic paralysis and even incurring huge losses of life and property. Since 2000, there have been six severe waterlogging events (waterlogging depth $\geq 0.50 \mathrm{~m}$ and its duration $\geq 0.50$ hour: Wang, 2014; Zhou, 2016), i.e., July $10^{\text {th }}, 2004$; July $30^{\text {th }}$, 2006; August $1^{\text {st }}, 2007$; June $23^{\text {th }}$, 
2011; July $21^{\text {th }}, 2012$ and July $19^{\text {th }}, 2016$. The maximum daily precipitation is $162 \mathrm{~mm}, 117 \mathrm{~mm}$, $171 \mathrm{~mm}, 215 \mathrm{~mm}, 460 \mathrm{~mm}$ and $263 \mathrm{~mm}$, respectively, all of which are much greater than the torrential precipitation intensity $(50 \mathrm{~mm} /$ day $)$.

\subsection{Data sources}

The regional precipitation discrepancy is estimated by comparing observations at urban weather stations with those at surrounding rural weather stations. The long-term daily precipitation and temperature observations from 1975 to 2015 at 20 weather stations are obtained from the China meteorological administration (Table 1). There are four weather stations in the urban area (Chaoyang, Fengtai, Haidian and Shijingshan), nine weather stations in the inner suburb (Beijing, Changping, Daxing, Fangshan, Mentougou, Shunyi, Tongzhou, Xiayunling and Zhaitang) and seven weather stations in the outer suburb (Foyeding, Huairou, Miyun, Pinggu, Shangdianzi, Tanghekou and Yanqing), and all these weather stations cover the entire Beijing metropolitan region. The data set is subjected to strict quality control procedures.

Land use data are used to investigate the impact of urban expansion on local climate. In this study, the annual land use maps in the Beijing metropolitan region (30 $\mathrm{m} \times 30 \mathrm{~m}$ resolution) are collected from the Data Center for Resource and Environmental Sciences, Chinese Academy of Sciences (http://www.resdc.cn/). Eight distinct land use types are reclassified using the ArcGIS platform (ESRI, 2011) following the current land use classification of China (GBT 21010-2007) (China's national standard, 2007): paddy, dry farmland, bush, forest, grass, water, built-up area and unused land. The land use data were usually released every 10 years (i.e., 1980s, 1990s, 2000s and 2010s) in the past and were changed to be released every 5 years (i.e., 2015) (Liu et al., 2014). Thus, five periods of land use data (i.e., 1980s, 1990s, 2000s, 2010s and 2015) are collected.

\subsection{Methodology}

\subsubsection{Metrics of extreme precipitation and urbanization}

Traditional metrics related to precipitation intensity are deficient in covering the entirety of extreme precipitation (Karl and Knight, 1998). In addition to the precipitation intensity, other metrics are considered (i.e., magnitude, frequency, duration and timing) in our study and nine related metrics are adopted, including five magnitude metrics (PI, Pmx, P5days, P95 and P99), two frequency metrics (Days50 and Days25), one duration metric (CWD) and one timing metric (DatPmx). The descriptions and calculation procedures of all the metrics are summarized in Table 2. 
Urbanization is mainly manifested in physical and social characteristics, such as the expansion of urban areas, and the increase of built-up areas for physical characteristics, and the increase of urban population and aggregation for social characteristics. In our study, we only explore the relationships between physical metrics of urbanization processes and extreme precipitation metrics. Five landscape metrics are adopted to characterize the physical metrics, i.e., the ratio of urban area (UR, \%), the complexity of urbanization (urban edge density: ED, $\mathrm{m} /$ hectare; landscape shape index: LS), the fragmentation of urbanization (patch density: PD, unit/ hectare), and the dominance of urbanization (Shannon's evenness: SE) which is adverse to the diversity of urbanization. The descriptions and equations of all the landscape metrics are shown in Table 3 according to Maurer and McGill (2011). Three concentric buffers with radii of $3 \mathrm{~km}, 10 \mathrm{~km}$ and $20 \mathrm{~km}$ are established around each station. All landscape metrics are calculated for each buffer using Fragstats software (version 4.2) (McGarigal et al., 2012). Moreover, the weather conditions in the surrounding area (i.e., the other 19 weather stations) are considered because they also affect urban extreme precipitation and are not involved in all the selected physical metrics of urbanization. Two related metrics are adopted, i.e., $\mathrm{TN}\left({ }^{\circ} \mathrm{C}\right)$, which represents the average temperature in the surrounding area, and PN ( $\mathrm{mm})$, which is the average precipitation in the surrounding area.

\subsubsection{Temporal variation detection for extreme precipitation}

The trend test is a useful and popular way to statistically detect temporal variations in longterm series. In this study, the seasonal Mann-Kendall (MK) test (Hirsh and Slack, 1984), a nonparametric method, is applied to test the annual trends of all the metrics from 1975 to 2015. A positive value of standardized MK statistic $(Z)$ indicates that an increasing trend is detected for a metric series, while a negative $Z$ value indicates that a decreasing trend is detected. In the confidence interval of $\alpha$, if $|Z|>Z_{\alpha}$, then the existing trend is considered to be statistically significant. In this study, $\alpha$ is set to $95 \%$ with the corresponding $Z_{\alpha}$ of 1.96 . Moreover, the annual variation is estimated by a trend slope, which is calculated as:

$$
S L=\sum_{i=1}^{n} \sum_{j=i+1}^{n}\left[\frac{1}{n \cdot(n-i)} \cdot \frac{X_{i}-X_{j}}{i-j}\right],
$$

where $S L$ is the trend slope; $X_{i}$ and $X_{j}$ are the $i^{\text {th }}$ and $j^{\text {th }}$ values of metric series, respectively; $n$ is the length of metric series. 


\subsubsection{Regional distribution classification}

It is rather complicated to identify the regional variation characteristics of extreme precipitation by investigating all the metric discrepancies station by station. Regional classification is a sensible approach to identifying typical homogeneous features from numerous metrics and weather stations across a broad range of spatial scales (Huang and Ferng, 1990). This approach is widely used in environmental flow assessments (Arthington, 2012; Poff et al., 2010) and impact assessments of dam regulation or climate change (Mackey et al., 2012; Zhang et al., 2012, 2015a, b).

Therefore, cluster analysis is adopted to partition nine metrics of extreme precipitation at 20 weather stations into relatively independent groups, which can cover the regional distribution characteristics of extreme precipitation in the Beijing metropolitan area. Principal component analysis (PCA) is also used to eliminate multicollinearity among different metrics and to combine all the correlated metrics into independent factors. The cumulative variances of independent factors are set to over $95 \%$ in our study. A hierarchical cluster is employed for cluster analysis, in which the Euclidean distance (ED) is used to calculate the similarities of independent factors between any two weather stations, and Ward's method is used to minimize the sum of the Euclidean squared distance of any two clusters. The Goodman-Kruskal index (Goodman and Kruskal, 1954), the C index (Hubert and Schultz, 1976), and minimum cluster size are used to assess cluster performance (Zhang et al., 2015a). All analyses are implemented in R software (version 3.1.1) (R Development Core Team, 2010).

\subsubsection{Causal relationship detection}

Although correlation analysis (e.g., the Pearson or Kendall test) is able to detect the statistical relationship between independent and dependent variable series, it is deficient in interpreting the causal relationship (Kaufmann et al. 2007). In our study, the Granger causality method was used to investigate the relationship between extreme precipitation and multiple physical metrics of urbanization. The unrestricted model (Eq.2) and restricted model (Eq.3) of the Granger causality equations are formulated as follows:

$$
\begin{aligned}
P_{i, y, l}= & \alpha_{0}+\sum_{k=1}^{n} \alpha_{1, k} \cdot U I_{i, y-\operatorname{lag} U_{k}, k}+\alpha_{2} \cdot P_{i, y-\operatorname{lag} P, l}+\alpha_{3} \cdot T_{i, y} \\
& +\alpha_{4} \cdot\left(\frac{1}{N-1} \sum_{j=1, j \neq i}^{N} P_{j, y, l}\right)
\end{aligned}
$$




$$
\begin{aligned}
P_{i, y, l}= & \alpha_{0}+\alpha_{2} \cdot P_{i, y-\operatorname{lag} P, l}+\alpha_{3} \cdot T_{i, y} \\
& +\alpha_{4} \cdot\left(\frac{1}{N-1} \sum_{j=1, j \neq i}^{N} P_{j, y, l}\right),
\end{aligned}
$$

where $P_{i, y, l}$ and $U I_{i, y, k}$ are the $l^{\text {th }}$ metrics of extreme precipitation and the $k^{\text {th }}$ urbanization metrics at station $i$ at year $y ; T$ is the average temperature, which is able to indicate the $\mathrm{UHI}\left({ }^{\circ} \mathrm{O}\right) ; N$ and $n$ are the numbers of weather stations and urbanization metrics ( $N=20$ and $n=7$ in our study), respectively; $\alpha_{0}$ is the intercept and $\alpha_{1, k}, \alpha_{2}, \alpha_{3}$, and $\alpha_{4}$ are the regression coefficients of the $k^{\text {th }}$ urbanization metric, lagged precipitation, average temperature and precipitation in the surrounding area $(T N$ and $P N)$, respectively; $\operatorname{lag} U_{k}$ and $\operatorname{lag} P$ are the lag numbers for the $k^{\text {th }}$ urbanization metric and precipitation. Both $\operatorname{lag} U_{k}$ and $\operatorname{lag} P$ are set to be two years because only five periods of land use data are collected in our study (Kaufmann et al., 2007).

In the Granger causality test, the null hypothesis $\mathrm{H} 0$ is that $\alpha_{0}=\alpha_{1, k}=\alpha_{2}=\alpha_{3}=\alpha_{4}=0$ $(k=1,2, \ldots, \mathrm{n})$. The test statistic $\mathrm{F}$ is formulated as:

$$
F=\frac{\left(R S S_{r}-R S S_{u}\right) / \operatorname{lag} U}{R S S_{u} /(N-\operatorname{lag} U-\operatorname{lag} P-1)},
$$

where $R S S_{u}$ and $R S S_{r}$ are the residual sums of squares of the unrestricted model and restricted model, respectively. The test statistic $\mathrm{F}$ follows $\mathrm{F}$ distribution with the degree of freedom being $\operatorname{lag} U$ and $(N$-lag $U$-lagP $)$. If $F>F_{\alpha}(q, n-p-q-q)$, hypothesis $\mathrm{H} 0$ is rejected; thus extreme precipitation is causally related to the urbanization. Conversely, if hypothesis $\mathrm{H} 0$ is accepted, then extreme precipitation has no causal relationship with urbanization. In this study, $\alpha$ is set to $90 \%$ with a corresponding $F_{\alpha}$ of 1.65 due to the limited sample number (i.e., 20 stations) according to Kaufmann et al. (2007).

The Granger causality between the metrics of extreme precipitation $(n=9)$ and urbanization metrics $(\mathrm{n}=6)$ or weather metrics $(\mathrm{n}=2)$ are detected individually. The total number of detection times for each concentric buffer is 72 , i.e., $9 \times(6+2)$. The regression coefficients are estimated by ordinary least squares. All analyses are also implemented in R software (version 3.1.1) (R Development Core Team, 2010).

Furthermore, a regional climate model (Weather Research and Forecasting model: WRF) is adopted to elaborate on the potential physical impacts of urbanization on extreme precipitation. WRF is a next-generation mesoscale numerical weather prediction system designed for both atmospheric research and operational forecasting applications (Skamarock et al., 2008; Yang et al., 2014). The version used in our study is Advanced Research WRF(ARW) 3.9.1.1, downloaded from the website (http://www2.mmm.ucar.edu/wrf/users/). In our study, two domains are utilized with horizontal resolutions of $3 \mathrm{~km}$ and $1 \mathrm{~km}$. The larger domain covers Beijing city and the surrounding areas with 160 grids in the west-east direction and 180 grids in 
the south-north direction. The inner domain focuses on the Beijing metropolitan area with 181 grids in the west-east direction and 181 grids in the south-north direction. The time steps are 15 seconds. Due to the high temporal-spatial resolution, only two representative land use data (i.e., 1980s and 2015) are adopted and used as the static data in the WRF simulations to investigate the relationship between land use change and precipitation. Each simulation runs for two years (i.e., 2013 and 2014), and the initial and boundary conditions come from the ERA-interim reanalysis data with horizontal resolution of 0.75 degrees. The other model settings (e.g., domain sizes, time steps, SST update intervals) in these two simulations are exactly the same to ensure that the changes in precipitation are only caused by the change in land use. Several important atmospheric variables are produced under these two land use scenarios during torrential precipitation, including wind, pressure and air temperature, all of which are critical determinants related to precipitation.

\section{Results}

3.1. Spatial and temporal variations of extreme precipitation metrics

\subsubsection{Magnitude}

All the magnitude metrics of extreme precipitation show obvious regional discrepancies (Figure 2). The values of all the magnitude metrics in the urban area and inner suburb are always higher than those in the outer suburb (Table A1). The regional discrepancies of Pmx and P5days are the most obvious. For example, the annual average values of P5days are $113.96 \mathrm{~mm}, 116.18$ $\mathrm{mm}$ and $104.53 \mathrm{~mm}$ in the urban area, inner and outer suburbs, respectively. For the temporal variations, the magnitude metrics at most weather stations (70\% 95\% of total) decrease slightly during the period from 1975 to 2015 . Among these weather stations, there are only two stations (i.e., Huairou and Shangdianzi in the outer suburb) for PI, one station (i.e., Shangdianzi in the outer suburb) for Pmx and P99, and four stations (i.e., Daxing and Fangshan in the inner suburb, Pinggu and Shangdianzi in the outer suburb) for P5days whose temporal trends are statistically significant. Thus, the magnitude metrics in the outer suburb decrease most significantly, particularly at Shangdianzi. Moreover, the metrics at the remaining 5\% 30\% of the total weather stations increase slightly without statistically significant trends, and most of these weather stations are at the urban area and inner suburb, particularly for Pmx.

\subsubsection{Frequency}

The annual average days of both torrential rain (Days50) and heavy rain (Days25) present decrease gradually from the city center to the suburb, and decrease interannually at the station 
scale (Figure 3a,b and Table A1). The annual average days of Days50 are 1.79 days, 1.53 days and 1.30 days in the urban area, inner and outer suburbs, respectively. The annual average days of Days 25 are much greater than those of Days50, i.e., 6.45 days, 5.67 days and 5.53 days in the urban area, inner and outer suburbs, respectively. For the temporal variations, the metrics at most weather stations (85\% $90 \%$ of total) also show decreasing trends, among which, Days 25 decreases significantly at only two weather stations (i.e., Fangshan and Mentougou in the inner suburb). Furthermore, the metrics at the remaining two or three weather stations increase slightly without statistically significant trends, and most of these weather stations are in the outer suburb.

\subsubsection{Duration and timing}

For the consecutive precipitation days (CRD), the annual average values in the outer suburb are the longest (i.e., $7.02 \sim 10.07$ days), followed by those in the urban area (i.e., $7.76 \sim 8.27$ days) and inner suburb (i.e., $5.56 \sim 9.10$ days) (Figure $4 a$ ). In addition, the decreasing trends are detected at 19 weather stations ( $95 \%$ of the total), among which the trends at eight weather stations (two in the urban area, four in the inner suburb and two in the outer suburb) are statistically significant. The CRD value increases slightly without a statistically significant trend at only Shunyi in the inner suburb.

For the timing metrics, the daily maximum precipitation always appears in late July (Figure $4 \mathrm{~b}$ ), and it usually occurs 5 10 days later in the urban area and inner suburb than in the outer suburb. The DatPmx values at eight weather stations ( $40 \%$ of the total) show decreasing trends without statistical significance. The values at the remaining 12 weather stations increase slightly, and the trend is statistically significant at only Daxing in the inner suburb.

\subsection{Regional classification}

According to the aforementioned regional diversities, three representative regions are further identified for the entire metrics of extreme precipitation. The first four PCA factors whose cumulative variances are $97.4 \%$ of total variances, are selected for classification. The cluster performance is the most robust if three regions are divided because the Goodman-Kruskal index is at the maximum (0.69), the $\mathrm{C}$ index is at the minimum (0.11) and the minimum cluster size is four.

Among the three regions, most metrics of extreme precipitation show the same regional distribution characteristics, except the magnitude metric (P95) and the duration metric (CRD). The regional distribution characteristics are that the metrics in Regions 2 and 3 are the greatest and least, respectively, and most metrics in Region 1 are close to those of Region 2 (Figure 5). For the magnitude metrics, the average Pmx shows the most regional discrepancies. The average values in Regions 2 and 1 are $84.49 \mathrm{~mm}$ and $77.48 \mathrm{~mm}$, which are $60.6 \%$ and $47.3 \%$ greater than 
those in Region 3 (52.61 mm), respectively. The average P95 shows the least regional discrepancies. Those average values are $9.51 \mathrm{~mm} /$ day in Region 2 and $8.10 \mathrm{~mm} /$ day in Region 1 , which are $24.3 \%$ and $5.9 \%$ greater than those in Region $3(7.65 \mathrm{~mm} /$ day $)$, respectively. For the frequency metrics, the average Days50 values are 1.78 days in Region 2 and 1.70 days in Region 1, which are extended 1.18 days and 1.10 days, respectively, compared to Region 3 (0.60 days), while the average Days25 values are 6.71 days in Region 2 and 6.09 days in Region 1, which are extended 2.96 days and 2.35 days, respectively, compared to Region 3. For the timing metrics, the average DatPmx values are July $27^{\text {th }}$ in Region 2 and $24^{\text {th }}$ in Region 1 , which are advanced seven days and four days more than those in Region $3\left(20^{\text {th }}\right)$, respectively.

The geographical and urbanization features also show obvious diversities among these three regions. There are 11 weather stations (four in the urban area and seven in the inner suburb) in Region 1 (Figure 6), all of which are located in the piedmont plain with high urbanization ratios; five weather stations (one in the inner suburb and four in the outer suburb) in Region 2, which are located in the windward slope of the topographic area; and four weather stations (two weather stations in the inner suburb and two weather stations in the outer suburb) in Region 3, which are located in the mountainous area with low urbanization ratios. Most of the urbanization metrics (i.e., UR, ED, LS, PD and TN) are always the largest in Region 1, followed by Region 2 and Region 3 (Figure 7). The average values in Region 1 are between $38.28 \%$ and $54.41 \%$ for UR, between $15.25 \mathrm{~m} / \mathrm{ha}$ and $20.23 \mathrm{~m} /$ ha for ED, between 4.99 and 23.98 for LS, between 0.79 $1 /$ ha and $1.171 /$ ha for PD, and $12.90^{\circ} \mathrm{C}$ for TN among the different concentric buffers. Additionally, the average SE is the largest in Region 2 (0.70 0.78), followed by Regions 3 (0.71 0.72) and $1(0.55 \sim 0.61)$, and the average PN is the largest in Region $2(12.23 \mathrm{~mm})$, followed by Regions $1(11.83 \mathrm{~mm})$ and $3(9.20 \mathrm{~mm})$. By the ANOSIM test, the regional differences of individual metrics are statistically significant in the $95 \%$ confidence interval (RANOSIM=0.09 0.76), except LS, PD, SE and TN (Table 4). The RANOSIM is 0.60 $(\mathrm{p}=0.001)$ for the geographical feature group and $0.62 \sim 0.86(\mathrm{p}=0.001$ or 0.002$)$ for the urbanization feature group. Specifically, the regional differences of the urban area are the most obvious (RANOSIM=0.68 0.76, $\mathrm{p}=0.001$ ), followed by the complexity of urbanization (RANOSIM=0.24 0.58, $\mathrm{p}<0.01$ ), the fragmentation of urbanization (RANOSIM=0.27 for $3 \mathrm{~km}$ buffer, $\mathrm{p}=0.02$ ) and the dominance of urbanization (RANOSIM=0.24 for $10 \mathrm{~km}$ buffer, $\mathrm{p}=0.03$ ). Along with the increasing buffer radius, the $\mathrm{p}$ values of the urbanization metrics become so large that the number of significant metrics in the $95 \%$ significance interval decreases gradually, particularly for LS, PD and SE.

Therefore, the urban precipitation island is identified mainly in the central urban region and the windward slope of the topographic area (Regions 1 and 2). The precipitation magnitudes in the urban precipitation island are much greater than those in the mountainous area in the outer suburb (Region 3). Both the torrential and heavy precipitation in the central urban region and windward slope (Regions 1 and 2) occur much more frequently and the daily maximum precipitation is usually delayed three or five days. Furthermore, along with the enhancements of 
extreme precipitation in Regions 1 and 2, the corresponding urbanization metrics are also much greater than those in the outer suburb (Region 3), particularly for UR, ED, TN and PN. For example, UR values in Regions 1 and 2 are 27.30\% 99.49\% and 2.47\% 50.76\%, respectively, while those in Region 3 are only 0.0 8.19\%. Therefore, Regions 1 and 2 are highly urbanized regions, while Region 3 is still rural. Thus, the urban signatures in the extreme precipitation metrics are explored mainly in Regions 1 and 2.

\subsection{Urban signatures}

\subsubsection{Causal relationships between extreme precipitation and urbanization}

For all the metrics of extreme precipitation, the metrics whose Granger relationships are significant with urbanization metrics show consistent variations among $3 \mathrm{~km}, 10 \mathrm{~km}$ and $20 \mathrm{~km}$ concentric buffers (Figure 8). The number of significant metrics is the greatest in Region 1, followed by Region 2 (Table A1). For the magnitude metrics, PI is the most significant because the Granger relationships with most urbanization metrics are statistically significant. In particular, all eight urbanization metrics are the Granger causes in Region 1. However, P95 is the least significant with only one to three Granger causes. For the frequency metrics, regional variations between Days25 and Days50 are not obvious, which are caused by six to eight, and two to four urbanization metrics in Regions 1 and 2, respectively. For the duration metrics, CRD is causally affected by five to six urbanization metrics in Region 1 and two to four metrics in Region 2. For the timing metrics, DatPmx is causally affected by precipitation in the surrounding area (PN) only in Region 1. Therefore, the significant metrics of extreme precipitation are PI in the magnitude group, Days25 and Days50 in the frequency group, and CRD in the duration group.

For all urbanization causes, the variation patterns among different buffers are also consistent. The most significant urbanization causes are in Region 1, followed by Region 2. For the area metrics (Figure 9), UR is the Granger cause of five precipitation metrics (i.e., PI and P99 in the magnitude metrics, Days50 and Days25 in the frequency metrics, and CRD in the duration metrics) in Region 1. However, no Granger relationships between UR and precipitation metrics are found in Region 2. For the complexity metrics, LS is the most significant cause of all the precipitation metrics in Region 1 (except P95 and DatPmx) and two metrics (i.e., PI and CRD) in Region 2. For the fragmentation metrics, PD is the most significant cause of all the precipitation metrics in Regions 1 and 2 (except DatPmx). For the dominance metrics, SE affects three to five precipitation metrics (i.e., PI, P99 and P95 in the magnitude metrics, Days50 and Days25 in the frequency metrics) in Region 1 and three to seven metrics except Days25 and DatPmx in Region 2. For the weather metrics, precipitation in the surrounding area $(\mathrm{PN})$ is the significant cause of all precipitation metrics (except DatPmx or CRD) in both Regions 1 and 2, and TN is significant only in Region 1. 


\subsubsection{Regression coefficients between extreme precipitation and urbanization}

Among all the three buffers, the number of significant coefficients in the $90 \%$ confidence interval is the greatest in Region 1, particularly for the magnitude metrics of extreme precipitation, followed by Region 2. In Region 1, most magnitude metrics except P95, have the Granger relationships with all urbanization and weather metrics. The detailed relationships are that urban area (UR), complexity (ED and LS) and fragmentation (PD), and weather metrics (TN and $\mathrm{PN}$ ) positively affect the magnitude metrics of extreme precipitation, while the effects of urbanization dominance (SE) are negative. For the frequency metrics (Days50 and Days25), the effects of urbanization and weather metrics are basically similar to those of the magnitude metrics. The effects of urban area (UR), complexity (ED and LS) and fragmentation (PD), and weather metrics (TN and PN) are positive, while those of urbanization dominance (SE) are negative. For the duration metrics (CRD), the effects of urban area (UR) and TN are negative, while those of complexity (LS), fragmentation (PD) and dominance (SE) are positive. For the timing metric (DatPmx), only the weather metric (PN) is a positive cause.

In Region 2, all the metrics of extreme precipitation, except DatPmx are affected significantly by the urbanization or weather metrics. In particular, the cause numbers for PI and CRD are much greater. For the magnitude metrics, the positive causes are the urbanization complexity (ED and LS) for PI, and fragmentation (PD) for all the magnitude metrics except Pmx, while the negative causes are the urbanization dominance (SE) for PI, Pmx and P5days. For the frequency metrics, the weather metric (PN) has a negative effect on Days50 while PD in fragmentation has a positive effect on Days25. For the duration metrics (CRD), some positive causes are detected including urban area (UR), complexity (ED and LS), fragmentation (PD), and PN in the weather metrics. Moreover, no causes are detected for the timing metric (DatPmx).

\subsection{Underlying causal mechanisms of urbanization by WRF simulations}

The urban heat island is one of the main driving factors for convective precipitation. The average air temperatures in the urban regions (Regions 1 and 2) are much greater than those in the mountainous region (Region 3 ) according to the station records and the WRF simulations (Figure 10a, d). The difference is nearly $4{ }^{\circ} \mathrm{C}$ from 1975 to 2015 according to the observations. Furthermore, except some outlier grids, the air temperature in most grids of Regions 1 and 2 increases nearly $0.0 \sim 0.5^{\circ} \mathrm{C}$ when the land use changes from the $1980 \mathrm{~s}$ to 2015 , and decreases over $0.5^{\circ} \mathrm{C}$ in Region 3 (Figure $10 \mathrm{~g}$ ). Therefore, the urban heat island is most likely more obvious along with the consistent expansion of impervious surface area from the 1980s to 2015.

The pressure field is highly related to convective intensity. Its regional difference is also quite obvious and in a good agreement with that of air temperature under the land use scenarios 
of 1980s and 2015 (Figure 10b, e) . By comparing the differences between these two scenarios, the air pressure during torrential precipitation decreases remarkably in the mountainous region, but appears to increase slightly in Regions 1 and 2. The air pressure differences between the urban regions and the mountainous region are much greater under the 2015 land use scenario of than those under the 1980s land use scenario (Figure 10h). Therefore, the convective intensity is probably strengthened during torrential precipitation due to urbanization from the 1980s to 2015 .

As the channel for water vapor transport, the wind field is also simulated under different land use scenarios (1980s and 2015) during torrential precipitation (Figure 10c, f). The simulations show that the Beijing metropolitan region is generally influenced by the southeasterly wind, which coincides well with the regional characteristics of the East Asia summer monsoon in China, but is not consistent with the findings reported by Yang et al. (2014), i.e., southwesterly wind. Abundant water vapor transferred with the southeasterly summer monsoon wind meets the urbanization-induced convections in the Beijing metropolitan area, making convective precipitation events occur more easily. Comparing the wind differences between the land use scenarios of the 1980s and 2015 (Figure 10i), much more water vapor will be transferred to Regions 1 and 2, but the differences are not obvious in Region 3. Thus, the intensity and frequency of convective precipitation are enhanced in the urban area (i.e., Regions 1 and 2) under the 2015 land use scenario. More water vapor flux is provided by a high rate of evaporation on the impervious surfaces, particularly prior to precipitation events, which is also of benefit to convergence (Ramamurthy and Bou-Zeid, 2014). Furthermore, topographic relief is another critical cause of extreme precipitation (Shepherd, 2005). The simulated wind field passes through the junction regions between mountainous and urban areas from the southwest to the northeast of the Beijing metropolitan area, and the wind speed decreases due to topographic uplift. Thus, more topographic precipitation would also be caused. The extreme precipitation metrics in Region 2 are probably enhanced by the combined effects of topographic relief and urbanization. These findings are consistent with the results of Yang et al. (2014).

For the duration and timing of extreme precipitation, the metrics are extended along with the expansions of the urban area and its dominance, and inversely related to urbanization complexity, fragmentation and the heat island in our study region. However, these further urbanization-induced mechanisms need more investigation with more mathematical experiments with longer simulation periods. Ensemble runs are also required to reduce the model uncertainties. However, this would be rather time-consuming and costly at the current stage.

\section{Discussion}

Our paper investigates the underlying causal mechanism of urbanization on regional patterns of extreme precipitation in the Beijing metropolitan area in a comprehensive manner. The main objectives focus on the entirety of extreme precipitation characterized by magnitude, frequency, duration and timing metrics, instead of the traditional precipitation intensity (Emmanuel et al., 
2012; Jauregui and Romales, 1996; Smith et al., 2012; Song et al., 2014; Yang et al., 2012). Multiple physical metrics of urbanization are also considered in detail by landscape and weather metrics, including complexity, fragmentation, dominance, temperature and precipitation in the surrounding areas, in addition to traditional single area expansion. Both the landscape and weather metrics are deduced from the long-term historic data series from 1975 to 2015 at 20 stations. Thus, the assemblage of the weather and land use data is quite abundant to support the Granger causality test between urbanization and extreme precipitation. The detected causal relationships are further supported by the physical mechanism of regional climate modelling under different scenarios of land use changes, which makes the findings even more convincing. Therefore, our study is a further extension of existing studies (Kaufmann et al., 2007; Song et al., 2014; Yang et al., 2012).

Although most of the extreme precipitation metrics at all the weather stations do not show significant trends throughout the period from 1975 to 2015, most the metrics show obvious regional patterns at the average state, except some magnitude metrics with significant decrease trends in the outer suburb (e.g., PI, Pmx and P5days). The temporal variations of extreme precipitation metrics are generally consistent with Song et al. (2014) in consideration of the study period (1950 2012). Although a slight increase of total summer precipitation is found from 2008 to 2012 in the same study area by Yang et al. (2014), these changes are probably caused by the interannual fluctuation and are hard to pass the statistical trend test due to the short period (only five years). Thus, some future works could be strengthened, such the interdecadal variations of extreme precipitation metrics and the impact of dataset length on the temporal trend test. The urban precipitation island is identified in the central urban region and the windward slope, which seems to be more reasonable than previous studies in the Beijing metropolitan area (Song et al., 2014; Yang et al., 2014) due to more characteristics of extreme precipitation being considered. Urbanization affects not only the magnitudes and frequencies of extreme precipitation but also their durations and timings. The effects are obvious in the urban precipitation island, particularly in the central urban region due to the high percentages of impervious surface areas $(55.1 \% \sim 71.1 \%)$. Along with the expansion of the urban area and its landscape dominance, both magnitudes and frequencies of extreme precipitation are significantly increased, while the consecutive precipitation days are significantly decreased. Moreover, with increasing urbanization complexity and fragmentation, the magnitudes, frequencies and consecutive precipitation days are significantly increased. The urban heat island also increases the magnitudes and frequencies of extreme precipitation and extends the consecutive precipitation days in highly urbanized regions, particularly for the central urban region. The average precipitation in the surrounding areas shows different regional effects, which are the positive cause of extreme precipitation in the central urban region, but the negative cause in the windward slope. It should be noted that only the average precipitation in the surrounding areas is significant on the Julian date of the daily maximum precipitation.

These findings agree well with previous studies in Mexico City (Jauregui and Romales, 1996), New York City (Bornstein and Lin, 2000), Chicago (Changnon and Westcott, 2002) and 
cities of southeast China (Zhou et al., 2004). The underlying urbanization mechanisms are deduced: along with the expansion of urban areas and their landscapes dominance, thermodynamic and moisture stratification are severely altered at the mesoscale scale through the creation of urban heat islands, e.g., increasing surface albedos and storing and converting more heat energy in urban areas (Ramanathan et al., 2001; Shepherd, 2005; Ramamurthy and BouZeid, 2014). The urbanization-induced convections are enhanced due to the urban heat island (Shepherd, 2005). As the southeast monsoon with abundant water vapor fluxes meets the urban heat island, convective precipitation is prone to occur. Furthermore, the surface roughness increases due to the increase of urban fragmentation and complexity, which probably increases the wind turbulence and further enhances local convergence (Bornstein and Lin 2000; Changnon et al., 2002). Accordingly, along with the expansion of urban areas, the wind field in the central urban area is obviously strengthened due to turbulence, even though the wind intensity of the entire monsoon does not change. Although the aerosol impact is not considered in the current version of the WRF, at the local scale, more aerosols with high concentrations of cloud condensation nuclei are released by exhaust emissions of vehicles, commercial and residential buildings, which are positive for nucleating small cloud droplets (Kaufmann et al., 2007; Ramanathan et al., 2001; Sailor, 2010).

Most of these deductions have been investigated using surface observations and climate models (e.g., Kalnay and Cai, 2003; Kaufmann et al., 2007; Song et al., 2014; Yang et al., 2014; Bounoua et al., 2015; and a detailed review in Shepherd, 2004), and are also interpreted by the WRF simulations under different land use scenarios in our study, particularly for the causal mechanism of urbanization on the magnitude and frequency of extreme precipitation. However, the causal mechanism on the duration and timing of extreme precipitation is still not clarified in detail given the heavy calculation burden of WRF simulations and the limited representations of microphysical processes (e.g., aerosol and land surface properties) in the modelling approaches (Shepherd, 2004; Yang et al., 2014). Further efforts should be implemented by long-term simulations of regional climate models in consideration of complicated land surface processes, regional atmospheric circulations, aerosol-cloud interactions, anthropogenic heat release, etc. Additionally, climate change also plays an important role in precipitation changes at large scales, which are not considered in our study according to the findings of Zhao et al (2018) (i.e., the interdecadal change of precipitation is consistent with land use and land cover changes in the Beijing-Tianjin-Hebei Region). Nevertheless, the impacts of climate change or other teleconnections should be considered in the future work.

\section{Conclusions}

The regional variation patterns of extreme precipitation metrics (e.g., magnitude, frequency, duration and timing) are tested and clustered from 1975 to 2015 at 20 weather stations in the Beijing metropolitan area. Moreover, the urban signatures in extreme precipitation variations are investigated by Granger causal relationship detection and a process based modeling approach in 
the consideration of multiple physical metrics of urbanization, including area, complexity, fragmentation and dominance. The following conclusions are made as follows.

(1). At most stations (60\% 95\% of the total), the magnitude and frequency metrics of extreme precipitation decrease slightly throughout the whole period, the consecutive precipitation days are extended, and the Julian dates of daily maximum precipitation are delayed. Among these stations, only a few stations (0 40\% of the total) show statistically significant trends in the $95 \%$ confidence interval, and most of them are in the outer suburb for the magnitude metrics and in the inner suburb for the frequency, duration and timing metrics.

(2). Three statistically significant regions are clustered for the spatial discrepancies of extreme precipitation events, i.e., the central urban region, the windward region of the topographic area and the mountainous region. The urban precipitation island is identified in the windward region, where all the metrics are largest among all the regions, except the duration. The average values are $2.4 \% \sim 14.8 \%$ and $10.3 \% \sim 37.7 \%$ greater than those of the central urban region and mountainous region, respectively, for the magnitude; and $4.5 \% \sim 9.2 \%$ and $44.2 \% \sim 66.3 \%$ greater than those of the central urban region and the mountainous region respectively, for the frequency. Moreover, the average Julian dates of daily maximum precipitation are three and seven days later than those of the central urban region and mountainous region, respectively.

(3) The expansion of the urban area and its dominance among all the landscape classes significantly enhance both the magnitude and frequency of extreme precipitation events, but decrease their consecutive days. Additionally, the increasing irregularity, number of urban patches and urban heat islands significantly reinforce the magnitude and frequency and extend the consecutive days in all regions, particularly for the central urban region. The potential physical mechanisms revealed by the WRF simulations under different landuse scenarios are that the expansion of impervious surfaces causes the enhancement of regional differences in air temperature and pressure between the urban area and outer suburb, and thus, the convective intensity is strengthened. If the abundant water vapor fluxes transferred with the southeast monsoon reach the urban area, the fluxes ascend with the urbanization-induced convections and easily form the convective precipitation events. The topographic relief further promotes the magnitude and frequency of extreme precipitation.

The regional variation patterns of extreme precipitation characteristics and further causal mechanisms of urbanization will be beneficial to flood control and disaster mitigation management in metropolitan areas with high population densities. Along with the considerable boosting of extreme precipitation events, particularly for their magnitudes, frequencies and durations in the critical regions, urban disasters, such as torrential flooding and urban waterlogging are expected to be further strengthened. Scientific urbanization planning and construction should attract attention to alleviate the consequences of urbanization-induced convective precipitation, particularly in large cities. 


\section{Acknowledgments}

This study was supported by the Natural Science Foundation of China (No. 41671024), the Program for "Bingwei" Excellent Talents in IGSNRR, CAS (No. 2015RC201) and the International Fellowship Initiative, IGSNRR, CAS (No.2017VP04). Thanks to the Data Center of Resources and Environmental Science, Chinese Academy of Sciences (http://www.resdc.cn/) for offering the GIS data and to the China Meteorological Data Sharing Service System (http://cdc.cma.gov.cn/home.do) for offering historical meteorological observations. Thanks to the editor and three anonymous reviewers for their insightful comments.

\section{References}

Antoine, L. \& Sylvain, J. (2016). Using amap and ctc packages for huge clustering. R News. 6, $58-60$.

Arthington, A.H. (2012). Environmental flows: saving rivers in the third millennium. University of California Press, Berkeley, CA. 406pp.

Beijing Municipal Bureau of Statistics (BMBS) and Survey Office of the National Bureau of Statistics in Beijing (SONBSB) (2015). Beijing Statistical Yearbook in 2015. China Statistical Press. (In Chinese)

Beijing Water Authority (BWA) (2015). Beijing Water Resources Bulletin of 2015. (In Chinese)

Bornstein, R. \& Lin, Q. (2000). Urban heat islands and summertime convective thunderstorms in Atlanta: three case studies. Atmospheric Environment, 34(3), 507-516. doi:10.1016/s13522310(99)00374-x.

Bounoua, L., Zhang, P., Mostovoy, G., Thome, K., Masek, J., Imhoff, M., Shepherd, M., Quattrochi, D., Santanello, J., Silva, J., Wolfe, R. \& Toure, A.M. (2015). Impact of urbanization on us surface climate. Environmental Research Letters, 10(8), 084010. doi:10.1088/17489326/10/8/084010.

Changnon, S. A. \& Westcott, N. E. (2002). Heavy rainstorms in Chicago: Increasing frequency, altered impacts, and future implications. Journal of the American Water Resources Association, 38(5), 1467-1475. doi:10.1111/j.1752-1688.2002.tb04359.x.

China's national standard (GB/T 21010-2007). (2007). Current land use classification. Beijing: China standards press.

Crutzen, P. J. (2004). New directions: The growing urban heat and pollution island effect-Impact on chemistry and climate. Atmospheric Environment, 38(21), 3539-3540. doi:10.1016/s13522310(04)00297-3. 
Emmanuel, I., Andrieu, H., Leblois, E. \& Flahaut, B. (2012). Temporal and spatial variability of rainfall at the urban hydrological scale. Journal of Hydrology, 430-431(6), 162-172. doi:10.1016/j.jhydrol.2012.02.013.

ESRI (Environmental Systems Research Institute) (2011). ESRI ArcGIS 10.0 Support Website. http://www.ESRI.com/10.0/webhelp.

Goodman, L. \& Kruskal, W. (1954). Measures of associations for cross-validations. Journal of the American Statistical Association, 49, 732-764.

Hirsh, R.M. \& Slack, J.R. (1984). A nonparametric trend test for seasonal data with serial dependence. Water Resources Research, 20(6), 727-732. doi:10.1029/wr020i006p00727.

Huang, S. \& Ferng, J. (1990). Applied land classification for surface water quality management: I. Watershed Classification. Journal of Environmental Management, 31(2), 107-126. doi:10.1016/s0301-4797(05)80002-7.

Hubert, L. \& Schultz, J. (1976). Quadratic assignment as a general data-analysis strategy. British Journal of Mathematical and Statistical Psychology, 29(2), 190-241. doi:10.1111/j.20448317.1976.tb00714.x.

Jauregui, E. \& Romales, E. (1996). Urban effects on convective precipitation in Mexico City. Atmospheric Environment, 30(20), 3383-3389. Doi: 10.1016/1352-2310(96)00041-6.

Kalnay, E. \& Cai, M. (2003). Impact of urbanization and land-use change on climate. Nature, 423(6939), 528-531. Doi: 10.1038/nature01675.

Karl, T. R. \& Knight, R. W. (1998). Secular trends of precipitation amount, frequency, and intensity in the United States. Bulletin of the American Meteorological Society, 79(2), 231-241. doi: 10.1175/1520-0477(1998)079<0231:STOPAF>2.0.CO;2.

Kaufmann, R. K., Seto, K. C., Schneider, A., Liu, Z., Zhou, L. \& Wang, W. (2007). Climate response to rapid urban growth: evidence of a human-induced precipitation deficit. Journal of Climate, 20(10), 2299-2306.doi: 10.1175/JCLI4109.1.

Liu, J.Y., Kuang, W.H., Zhang, Z.X., Xu, X.L., Qin, Y.W., Ning, J., Zhou, W.C., Zhang, S.W., Li, R.D., Yan, C.Z., Wu, S.X., Shi, X.Z., Jiang, N., Yu, D.S., Pan, X.Z., \& Chi, W.F. (2014). Spatiotemporal characteristics, patterns, and causes of land-use changes in China since the late 1980s. Journal of Geographical Sciences, 2014, 24(2):195-210..

Lowry, W. P. (1998). Urban effects on precipitation amount. Progress in Physical Geography, 22(4), 477-520. doi:10.1177/030913339802200403.

Mackey, C. W., Lee, X. \& Smith, R. B. (2012). Remotely sensing the cooling effects of city scale efforts to reduce urban heat island. Building and Environment, 49, 348-358.

doi:10.1016/j.buildenv.2011.08.004. 
Mardia, K. V., Kent, J. T. \& Bibby, J. M. (1979). Multivariate Analysis, London. Academic Press.

Maurer, B.A. \& McGill, B.J. (2011). Measurement of species diversity. In: Magurran, A.E., McGill, B.J. (Eds.), Biological Diversity: Frontiers in Measurement and Assessment. Oxford University Press, New York, USA pp. 55-65.

McGarigal, K., Cushman, S.A. \& Ene, E. (2012). FRAGSTATS v4: Spatial Pattern Analysis Program for Categorical and Continuous Maps. Computer software program produced by the authors at the University of Massachusetts, Amherst. Available at the following web site: http://www.umass.edu/landeco/research/fragstats/fragstats.html.

Meila, M. (2007). Comparing clustering --- An information based distance. Journal of Multivariate Analysis. 98(5), 873-895. doi:10.1016/j.jmva.2006.11.013.

Mölders, N. \& Olson, M. A. (2004). Impact of urban effects on precipitation in high latitudes. Journal of Hydrometeorology, 5(3), 409-429. doi:10.1175/1525-

7541(2004)005<0409:ioueop>2.0.co;2.

Oke, T. R. (1973). City size and the urban heat island. Atmospheric Environment, 7(8), 769-779. doi: 10.1016/0004-6981(73)90140-6.

Oke, T. R. (1982). The energetic basis of the urban heat island. Quarterly Journal of the Royal Meteorological Society, 108(455), 1-24. doi:10.1002/qj.49710845502.

Poff, N. L., Richter, B. D., Arthington, A. H., Bunn, S. E., Naiman, R. J. Kendy, E., Acreman, M., Apse, C., Bledsoe, B.P., Freeman, M.C., Henriksen, J., Jacobson, R.B., Kennen, J.G., Merritt, D.M., O'Keefe, J.H., Olden, J.D., Rogers, K., Tharme, R.E. \& Warner A. (2010). The ecological limits of hydrologic alteration (eloha): a new framework for developing regional environmental flow standards. Freshwater Biology, 55(1), 147-170. doi: 10.1111/j.13652427.2009.02204.x.

R Development Core Team. (2010). R: A language and environment for statistical computing. R Foundation for Statistical Computing, Vienna, Austria, ISBN 3-900051-07-0. http://www.Rproject.org.

Ramamurthy, P. \& Bou-Zeid, E. (2014). Contribution of impervious surfaces to urban evaporation. Water Resources Research, 50(4), 2889-2902. doi:10.1002/2013wr013909.

Ramanathan, V., Crutzen, P. J., Kiehl, J. T. \& Rosenfeld, D. (2001). Aerosols, Climate, and the Hydrological Cycle. Science, 294(5549), 2119-2124. doi:10.1126/science.1064034.

Ren, G.Y. \& Zhou, Y.Q. (2014). Urbanization effect on trends of extreme temperature indices of national stations over mainland China, 1961-2008. Journal of Climate, 27(6), 2340-2360.doi: 10.1175/jcli-d-13-00393.1. 
Sailor, D. J. (2010). A review of methods for estimating anthropogenic heat and moisture emissions in the urban environment. International Journal of Climatology, 31(2), 189-199. doi: 10.1002/joc. 2106.

Shepherd, J. M. (2005). A review of current investigations of urban-induced rainfall and recommendations for the future. Earth Interactions, 9(12), 1-27. doi: 10.1175/ei156.1.

Skamarock, W. C., Klemp, J. B., Dudhia, J., Gill, D. O., Barker, D. M., Duda, M. G., Huang, X.Y., Wang, W., and Powers, J. G. (2008), A description of the advanced research WRF version 3, Rep., 113 pp., National Center for Atmospheric Research, Boulder, Colorado, USA.

Smith, J. A., Baeck, M. L., Villarini, G., Welty, C., Miller, A. J. \& Krajewski, W. F. (2012). Analyses of a long-term, high-resolution radar rainfall data set for the Baltimore metropolitan region. Water Resources Research, 48(4), 4504. doi:10.1029/2011wr010641.

Song, X., Zhang, J., Aghakouchak, A., Roy, S. S., Xuan, Y., Wang, G., He, R.M., Wang, X.J. \& Liu, C.S. (2014). Rapid urbanization and changes in spatiotemporal characteristics of precipitation in Beijing metropolitan area. Journal of Geophysical Research Atmospheres, 119(19), 11,250-11,271. doi: 10.1002/2014JD022084.

Thielen, J., Wobrock, W., Gadian, A., Mestayer, P. G. \& Creutin, J. D. (2000). The possible influence of urban surfaces on rainfall development: A sensitivity study in 2D in the mesogamma scale. Atmospheric Research, 54(1), 15-39. doi:10.1016/s0169-8095(00)00041-7.

United Nations, Department of Economic and Social Affairs, Population Division. (2015). World Urbanization Prospects: The 2014 Revision. (ST/ESA/SER.A/366).New York.

Wang, J., Feng, J. \& Yan, Z. (2015). Potential sensitivity of warm season precipitation to urbanization extents: Modeling study in Beijing - Tianjin - Hebei urban agglomeration in China. Journal of Geophysical Research Atmospheres, 120(18), 9408-9425. doi: 10.1002/2015JD023572.

Wang, Q.(2014). Cause analysis and countermeasures of urban rainstorm waterlogging in Beijing. Water \& Wastewater Engineering, (S1),127-130. (in Chinese)

Xia, J., Zhang, Y. Y., Xiong, L. H., He, S., Wang, L. F. \& Yu, Z. B. (2017). Opportunities and challenges of the sponge city construction related to urban water issues in china. Science China Earth Sciences, 60(4), 652-658. doi:10.1007/s11430-016-0111-8.

Yang, L., Smith, J. A., Baeck, M. L., Bou-Zeid, E., Jessup, S. M., Tian, F. \& Hu, H. (2014). Impact of urbanization on heavy convective precipitation under strong large-scale forcing: A case study over the Milwaukee-Lake Michigan region. Journal of Hydrometeorology, 15(1), 261-278. doi: 10.1175/JHM-D-13-020.1.

Yang, L., Tian, F., Smith, J. A. \& Hu, H. (2014). Urban signatures in the spatial clustering of summer heavy rainfall events over the Beijing metropolitan region. Journal of Geophysical Research Atmospheres, 119(3), 1203-1217. doi: 10.1002/2013JD020762. 
Yang, Y. J., Wu, B. W., Shi, C. E., Zhang, J. H., Li, Y. B., Tang, W. A., Wen, H.Y., Zhang, H.Q. $\&$ Shi, T. (2012). Impacts of urbanization and station-relocation on surface air temperature series in Anhui Province, China. Pure and Applied Geophysics, 170(11), 1969-1983. doi:10.1007/s00024-012-0619-9.

Zeileis, A. \& Hothorn, T. (2002). Diagnostic Checking in Regression Relationships. R News. 2(3), 7-10. URL http://CRAN.R-project.org/doc/Rnews /.

Zhang, Y., Fu, G.B., Sun, B.Y., Zhang, S.F. \& Men, B.H. (2015a). Simulation and classification of the impacts of projected climate change on flow regimes in the arid Hexi Corridor of Northwest China. Journal of Geophysical Research: Atmospheres, 120(15), 7429-7453. doi:10.1002/2015jd023294.

Zhang, Y., Hong, Y., Wang, X., Gourley, J. J., Xue, X., Saharia, M., Ni, G.H., Wang, G.L., Huang, Y., Chen, S. \& Tang, G. Q. (2014). Hydrometeorological analysis and remote sensing of extremes: was the July 2012 Beijing flood event detectable and predictable by global satellite observing and global weather modeling systems? Journal of Hydrometeorology, 16(1), 381-395. DOI: 10.1175/JHM-D-14-0048.1.

Zhang, Y.Y., Arthington, A.H., Bunn, S.E., Mackay, S., Xia, J. \& Kennard, M. (2012). Classification of flow regimes for environmental flow assessment in regulated rivers: the Huai river basin, China. River Research and Applications, 28(7), 989-1005. doi:10.1002/rra.1483.

Zhang, Y.Y., Zhai, X.Y., Shao, Q.X. \& Yan, Z.Q. (2015b). Assessing temporal and spatial alterations of flow regimes in the regulated Huai River Basin, China. Journal of Hydrology, 529, 384-397. doi:10.1016/j.jhydrol.2015.08.001.

Zhao, L., Lee, X., Smith, R. B. \& Oleson, K. (2014). Strong contributions of local background climate to urban heat islands. Nature, 511(7508), 216-219, doi:10.1038/nature13462.

Zhao, N., Yue, T., Li, H., Zhang, L., Yin, X., \& Liu, Y. (2017). Spatio-temporal changes in precipitation over Beijing-Tianjin-Hebei region, China. Atmospheric Research, 202, 156-168.

Zhou L., Dickinson R., Tian Y.H., Fang J.Y., Li Q.X., Kaufmann R.K., Tucker, C.J. \& Myneni R.B. (2004). Evidence for a significant urbanization effect on climate in China. Proceedings of the National Academy of Sciences of the United States of America, 101(26), 9540-9544. doi: 10.1073/pnas.0400357101.

Zhou L.N. (2016). Research on risk identification of urban waterlogging based on SWMM, (Master's thesis). Retrieved from the Doctoral dissertation and master's thesis database. (http://epub.cnki.net/kns/default.htm). Chongqing, China: Southwest Jiaotong University (In Chinese). 
Tables

Table 1.

Selected Weather Stations and Their Surrounding Geophysical Attributes

\begin{tabular}{|clccc|}
\hline Name & Location & Ratio of urban area $(\%)$ & Elevation(m) & $\begin{array}{c}\text { Daily precipitation } \\
\text { intensity }(\mathrm{mm})\end{array}$ \\
Chaoyang & Urban area & 71.10 & 35.30 & 12.40 \\
Fengtai & Urban area & 66.03 & 55.20 & 12.19 \\
Haidian & Urban area & 66.76 & 45.80 & 12.56 \\
\hline Shijingshan & Urban area & 55.17 & 65.60 & 11.97 \\
Beijing & Inner suburb & 66.71 & 31.30 & 12.17 \\
Changping & Inner suburb & 27.30 & 76.20 & 11.23 \\
Daxing & Inner suburb & 52.44 & 37.60 & 12.09 \\
\hline Fangshan & Inner suburb & 43.09 & 39.20 & 11.86 \\
Mentougou & Inner suburb & 40.25 & 92.70 & 12.48 \\
\hline Shunyi & Inner suburb & 40.86 & 28.60 & 12.63 \\
Tongzhou & Inner suburb & 57.89 & 43.30 & 12.26 \\
\hline Xiayunling & Inner suburb & 8.19 & 407.70 & 11.92 \\
Zhaitang & Inner suburb & 2.93 & 440.30 & 9.18 \\
\hline Foyeding & Outer suburb & 5.33 & 1224.70 & 12.81 \\
Huairou & Outer suburb & 17.70 & 75.70 & 12.79 \\
\hline Miyun & Outer suburb & 14.07 & 71.80 & 13.11 \\
\hline Pinggu & Outer suburb & 14.81 & 32.10 & 11.73 \\
\hline Shangdianzi & Outer suburb & 4.28 & 293.30 & 9.48 \\
\hline Tanghekou & Outer suburb & 0.97 & 331.60 & 9.01 \\
\hline Yanqing & Outer suburb & 9.21 & 487.90 & $\mathrm{~b}$ \\
\hline
\end{tabular}

Note: Ratio of urban area is obtained based on the land use data of 2015 in $10 \mathrm{~km}$ buffer; Precipitation intensity is obtained based on the daily observed precipitation series from 1975 to 2015. 


\section{Table 2.}

Metrics Used for Description of Extreme Precipitation

\begin{tabular}{|clll|}
\hline Groups & Metrics & \multicolumn{2}{l|}{ Abbreviation Units } \\
& Annual precipitation amounts/total annual precipitation days & PI & $\mathrm{mm} /$ day \\
& Daily maximum precipitation amount & Pmx & $\mathrm{mm}$ \\
Magnitude & Maximum precipitation amount within five consecutive days & P5days & $\mathrm{mm}$ \\
& The 95th percentiles of daily precipitation & P95 & $\mathrm{mm}$ \\
& The 99th percentiles of daily precipitation & P99 & $\mathrm{mm}$ \\
Frequency & Annual days of torrential precipitation (PI is no less than $50 \mathrm{~mm} /$ day) & Days50 & days \\
Frequency & Annual days of heavy precipitation (PI is no less than $25 \mathrm{~mm} /$ day) & Days 25 & days \\
Duration & Consecutive precipitation days & CRD & days \\
Timing & Julian date of the daily maximum precipitation & DatPmx & none \\
\hline
\end{tabular}


Table 3.

Landscape Metrics Used for Description of Urbanization

\begin{tabular}{|c|c|c|c|c|}
\hline Groups & Landscape Metrics & Equations & Descriptions & Units \\
\hline Area & Ratio of urban area (UR) & $U R=\sum_{j=1}^{n} \alpha_{j} / A$ & $\begin{array}{l}\text { Expansion } \\
\text { of urban } \\
\text { area }\end{array}$ & $\%$ \\
\hline \multirow[b]{2}{*}{ Complexity } & Urban edge density (ED) & $E D=E / A$ & \multirow{2}{*}{$\begin{array}{c}\text { Irregularity } \\
\text { of urban } \\
\text { patches }\end{array}$} & $\begin{array}{c}\mathrm{m} / \\
\text { hectare }\end{array}$ \\
\hline & $\begin{array}{l}\text { Landscape shape index } \\
\text { (LS) }\end{array}$ & $L S=0.25 \cdot \sum_{k=1}^{m} e_{k} / \sqrt{A}$ & & none \\
\hline Fragmentation & Patch density (PD) & $P D=n / A$ & $\begin{array}{l}\text { Number of } \\
\text { urban } \\
\text { patches }\end{array}$ & $\begin{array}{c}1 / \\
\text { hectare }\end{array}$ \\
\hline Diversity & Shannon's evenness (SE) & $S E=-\left[\sum_{i=1}^{m}\left(P_{i} \cdot \ln P_{i}\right)\right] / \ln m$ & $\begin{array}{l}\text { Dominance } \\
\text { of urban } \\
\text { patches }\end{array}$ & none \\
\hline
\end{tabular}

Note: $a_{j}$ is area (hectare, i.e., $10^{4} \mathrm{~m}^{2}$ ) of urban land patch $j ; A$ is total landscape area (hectare); $E$ is the total edge length $(\mathrm{m})$ of urban patch class; $e_{k}$ is the total edge length $(\mathrm{m})$ between urban land patch and other patch class $k$, including the entire landscape boundary and some or all background edge segments involving urban land patch; $n$ is total patch number of urban land class; $P_{i}$ is proportion of the landscape occupied by patch $i ; m$ is total number of landscape classes. 


\section{Table 4.}

Similarity Test of Geographical, Urbanization and Weather Features among Different Regions by ANOSIM

\begin{tabular}{|c|c|c|c|c|c|}
\hline \multirow{2}{*}{\multicolumn{3}{|c|}{ Factor categories }} & \multirow{3}{*}{$\begin{array}{c}\text { Factors } \\
\text { Longitude }\end{array}$} & \multicolumn{2}{|c|}{$\mathrm{R}_{\text {ANOSIM }}$} \\
\hline & & & & For single factor & For factor category \\
\hline \multirow{3}{*}{\multicolumn{3}{|c|}{ Geography }} & & $0.50(p=0.002)$ & \multirow{3}{*}{$0.60(p=0.001)$} \\
\hline & & & Latitude & $0.52(p=0.002)$ & \\
\hline & & & Elevation & $0.58(p=0.002)$ & \\
\hline \multirow{15}{*}{ Urbanization } & \multirow{5}{*}{$\begin{array}{c}3 \mathrm{~km} \\
\text { buffer }\end{array}$} & Area & UR & $0.68(p=0.001)$ & \multirow{5}{*}{$0.62(p=0.002)$} \\
\hline & & \multirow{2}{*}{ Complexity } & ED & $0.24(p=0.030)$ & \\
\hline & & & $\mathrm{LS}$ & $0.13(\mathrm{p}=0.142)$ & \\
\hline & & Fragmentation & PD & $0.27(p=0.020)$ & \\
\hline & & Diversity & SE & $0.13(\mathrm{p}=0.128)$ & \\
\hline & \multirow{6}{*}{$\begin{array}{l}10 \mathrm{~km} \\
\text { buffer }\end{array}$} & Area & UR & $0.72(p=0.001)$ & \multirow{5}{*}{$0.86(p=0.001)$} \\
\hline & & \multirow{2}{*}{ Complexity } & ED & $0.58(p=0.001)$ & \\
\hline & & & $\mathrm{LS}$ & $0.12(\mathrm{p}=0.142)$ & \\
\hline & & Fragmentation & PD & $0.10(\mathrm{p}=0.177)$ & \\
\hline & & Diversity & SE & $0.24(p=0.032)$ & \\
\hline & & Area & UR & $0.76(p=0.001)$ & \multirow{5}{*}{$0.76(p=0.001)$} \\
\hline & \multirow{4}{*}{$\begin{array}{l}20 \mathrm{~km} \\
\text { buffer }\end{array}$} & \multirow{2}{*}{ Complexity } & ED & $0.35(p=0.006)$ & \\
\hline & & & LS & $0.13(\mathrm{p}=0.132)$ & \\
\hline & & Fragmentation & PD & $0.14(\mathrm{p}=0.125)$ & \\
\hline & & Diversity & SE & $0.09(\mathrm{p}=0.211)$ & \\
\hline \multirow{2}{*}{\multicolumn{3}{|c|}{ Weather }} & $\mathrm{TN}$ & $0.20(\mathrm{p}=0.075)$ & \multirow{2}{*}{$0.31(p=0.019)$} \\
\hline & & & PN & $0.52(p=0.005)$ & \\
\hline
\end{tabular}

Note. The bold number is the detection which is statistically significant (i.e., $\mathrm{p} \leq 0.05$ ) 
Confidential manuscript submitted to Journal of Geophysical Research: Atmosphere

\section{Appendix A}

\section{Table A1.}

Annual Average Values of Extreme Precipitation Metrics and Their Inter-annual Variations at All the 20 Stations

\begin{tabular}{|c|c|c|c|c|c|c|c|c|c|c|c|c|c|c|c|c|c|c|c|}
\hline \multirow{3}{*}{ Name } & \multirow{3}{*}{ Location } & \multicolumn{2}{|c|}{ PI } & \multicolumn{2}{|c|}{$\operatorname{Pmx}$} & \multicolumn{2}{|c|}{ P5days } & \multicolumn{2}{|c|}{ P95 } & \multicolumn{2}{|c|}{ P99 } & \multicolumn{2}{|c|}{ Days50 } & \multicolumn{2}{|c|}{ Days25 } & \multicolumn{2}{|c|}{ CRD } & \multicolumn{2}{|c|}{ DatPmx } \\
\hline & & mean & slope & mean & slope & mean & slope & mean & slope & mean & slope & mean & slope & mean & slope & mean & slope & mean & slope \\
\hline & & $\mathrm{mm} /$ day & $\begin{array}{c}\mathrm{mm} / \mathrm{day} \\
\text { /year }\end{array}$ & $\mathrm{mm}$ & $\mathrm{mm} /$ year & $\mathrm{mm}$ & $\mathrm{mm} /$ year & $\mathrm{mm}$ & $\mathrm{mm} /$ year & $\mathrm{mm}$ & $\mathrm{mm} /$ year & days & days/year & days & days/year & days & days/year & none & days/year \\
\hline Chaoyang & Urban area & 12.40 & -0.037 & 74.44 & -0.085 & 117.16 & -0.729 & 8.60 & -0.001 & 32.64 & -0.100 & 1.78 & -0.018 & 6.78 & -0.036 & 7.95 & -0.028 & 209.32 & 0.351 \\
\hline Fengtai & Urban area & 12.19 & -0.021 & 73.73 & 0.037 & 109.45 & -0.545 & 8.10 & -0.018 & 31.75 & -0.067 & 1.81 & 0.009 & 6.17 & -0.060 & 7.76 & -0.059 & 201.73 & -0.711 \\
\hline Haidian & Urban area & 12.56 & 0.003 & 76.40 & -0.098 & 117.80 & -0.322 & 8.33 & -0.010 & 33.50 & -0.067 & 1.98 & -0.006 & 6.56 & -0.016 & 8.20 & -0.140 & 206.27 & 0.394 \\
\hline Shijingshan & Urban area & 11.97 & -0.001 & 75.68 & 0.723 & 111.44 & 0.147 & 8.12 & -0.004 & 32.36 & -0.036 & 1.61 & -0.007 & 6.29 & -0.042 & 8.27 & -0.070 & 205.44 & 0.447 \\
\hline Beijing & Inner suburb & 12.17 & -0.040 & 70.00 & -0.443 & 113.49 & -1.222 & 7.88 & -0.024 & 32.42 & -0.165 & 1.71 & -0.029 & 6.27 & -0.089 & 5.56 & -0.025 & 207.66 & 0.310 \\
\hline Changping & Inner suburb & 11.23 & -0.021 & 79.36 & -0.092 & 115.22 & -0.433 & 7.41 & 0.003 & 29.39 & -0.089 & 1.44 & -0.004 & 4.81 & -0.052 & 9.10 & -0.030 & 204.02 & -0.044 \\
\hline Daxing & Inner suburb & 12.09 & -0.031 & 76.10 & -0.978 & 117.23 & -1.878 & 8.06 & -0.024 & 30.80 & -0.177 & 1.51 & -0.012 & 5.83 & -0.046 & 7.83 & -0.037 & 204.93 & 0.976 \\
\hline Fangshan & Inner suburb & 11.86 & -0.037 & 76.85 & -0.646 & 109.35 & -1.505 & 8.06 & -0.034 & 31.49 & -0.218 & 1.78 & -0.011 & 5.81 & -0.087 & 8.05 & -0.090 & 204.83 & -0.443 \\
\hline Mentougou & Inner suburb & 12.48 & -0.045 & 87.72 & 0.424 & 129.86 & -0.238 & 8.45 & -0.045 & 33.79 & -0.222 & 1.78 & -0.035 & 6.15 & -0.080 & 8.15 & -0.088 & 199.51 & 0.061 \\
\hline Shunyi & Inner suburb & 12.63 & -0.033 & 82.01 & 0.159 & 124.34 & -0.561 & 8.40 & -0.038 & 32.84 & -0.108 & 1.73 & -0.016 & 6.56 & -0.042 & 8.12 & 0.016 & 204.51 & 0.591 \\
\hline Tongzhou & Inner suburb & 12.26 & -0.034 & 79.95 & -0.502 & 121.99 & -1.272 & 7.72 & -0.019 & 31.80 & -0.144 & 1.61 & -0.035 & 5.81 & -0.026 & 7.76 & -0.058 & 201.07 & 0.640 \\
\hline Xiayunling & Inner suburb & 11.92 & -0.055 & 90.98 & -0.310 & 130.09 & -0.935 & 9.29 & -0.070 & 32.09 & -0.133 & 1.68 & -0.013 & 6.54 & -0.076 & 7.37 & -0.063 & 205.93 & -0.415 \\
\hline Zhaitang & Inner suburb & 9.18 & -0.016 & 51.65 & 0.039 & 84.09 & -0.086 & 7.60 & -0.051 & 22.38 & -0.061 & 0.54 & -0.002 & 3.29 & -0.045 & 8.88 & -0.167 & 198.61 & -0.108 \\
\hline Foyeding & Outer suburb & 9.34 & 0.022 & 56.62 & -0.090 & 84.00 & -0.406 & 8.35 & -0.024 & 26.39 & 0.049 & 0.76 & 0.005 & 4.34 & 0.022 & 8.66 & -0.101 & 200.59 & 0.725 \\
\hline Huairou & Outer suburb & 12.81 & -0.082 & 89.08 & -0.691 & 130.41 & -1.061 & 9.53 & -0.048 & 34.81 & -0.210 & 1.81 & -0.038 & 6.76 & -0.055 & 9.49 & -0.041 & 211.34 & -0.229 \\
\hline Miyun & Outer suburb & 12.79 & -0.035 & 85.31 & -0.324 & 125.45 & -0.669 & 9.54 & -0.048 & 34.77 & -0.197 & 1.98 & -0.023 & 6.85 & -0.042 & 10.07 & -0.048 & 211.22 & 0.160 \\
\hline Pinggu & Outer suburb & 13.11 & -0.043 & 82.18 & -0.454 & 125.14 & -1.198 & 9.82 & -0.053 & 34.81 & -0.197 & 1.88 & -0.005 & 7.07 & -0.048 & 8.32 & -0.019 & 205.73 & 0.566 \\
\hline Shangdianzi & Outer suburb & 11.73 & -0.078 & 74.92 & -1.496 & 114.14 & -1.870 & 9.40 & -0.071 & 32.34 & -0.281 & 1.54 & -0.032 & 6.32 & -0.105 & 7.66 & -0.036 & 204.51 & -0.128 \\
\hline Tanghekou & Outer suburb & 9.48 & -0.005 & 54.53 & -0.213 & 81.03 & -0.597 & 7.49 & -0.018 & 24.80 & -0.089 & 0.63 & -0.015 & 3.90 & -0.034 & 7.02 & -0.019 & 203.81 & -0.197 \\
\hline Yanqing & Outer suburb & 9.01 & 0.009 & 47.63 & 0.034 & 71.53 & -0.207 & 7.17 & -0.016 & 23.12 & 0.024 & 0.49 & 0.001 & 3.49 & 0.013 & 8.05 & -0.105 & 199.24 & 0.736 \\
\hline
\end{tabular}

This article is protected by copyright. All rights reserved. 
Note: The bold value in the slope column means that the variation trend of a certain metric is statistically significant in the $95 \%$ confidence interval.

\section{Table A2.}

Regression Coefficients Between Extreme Precipitation and Urbanization Estimated By Ordinary Least Squares in Regions 1 and 2 for Different Buffers

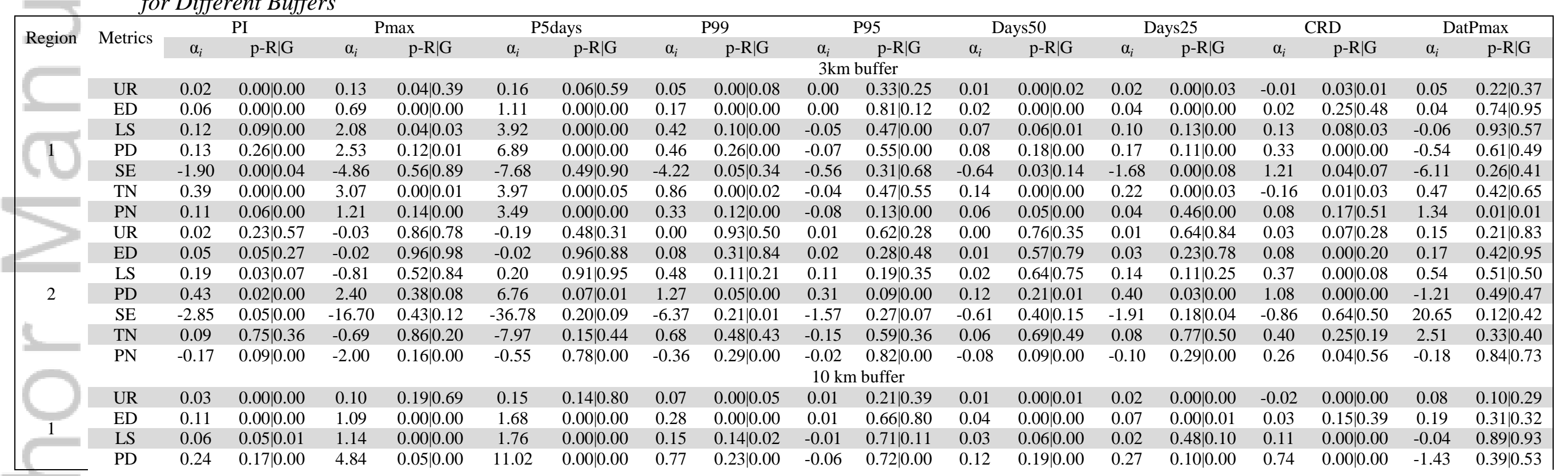

This article is protected by copyright. All rights reserved. 


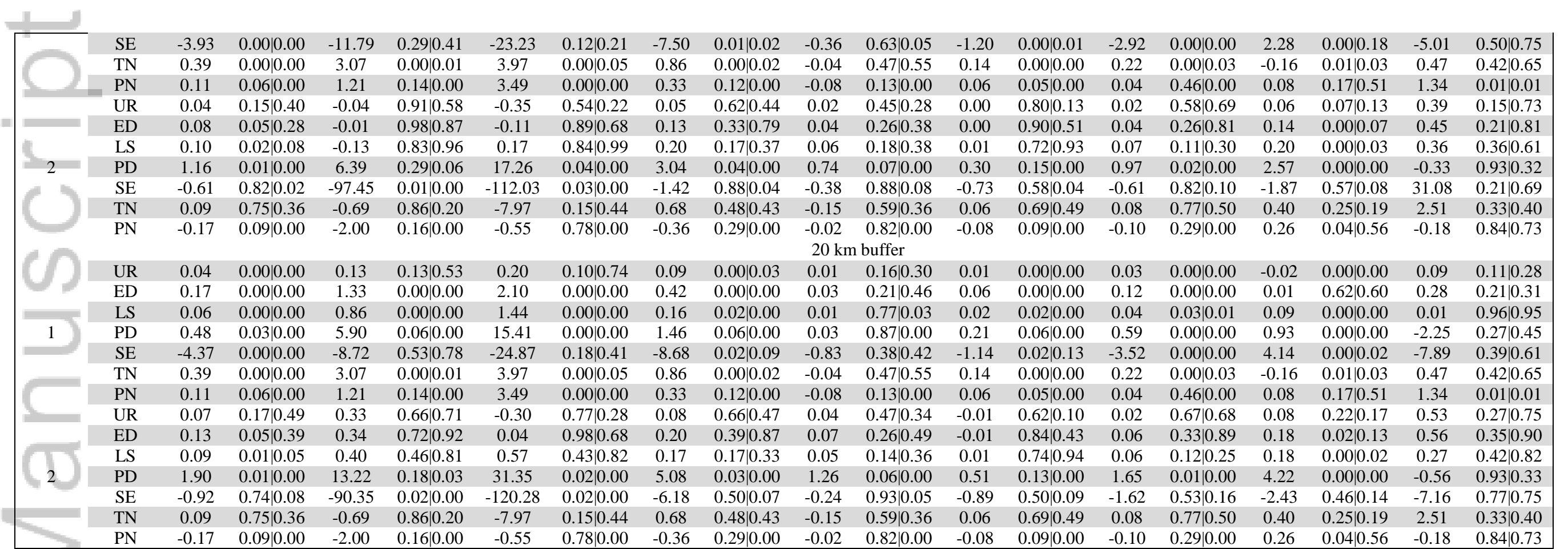

Note: $\alpha_{i}$ is the regression coefficient between extreme precipitation and urbanization, weather condition in the surrounding area,

$i=1,2, \ldots, 4 ; \mathrm{p}-\mathrm{R} \mid \mathrm{G}$ is the $\mathrm{p}$ values for both the regression coefficient analysis (left value) and casual relationship detection (right value), respectively.

This article is protected by copyright. All rights reserved. 


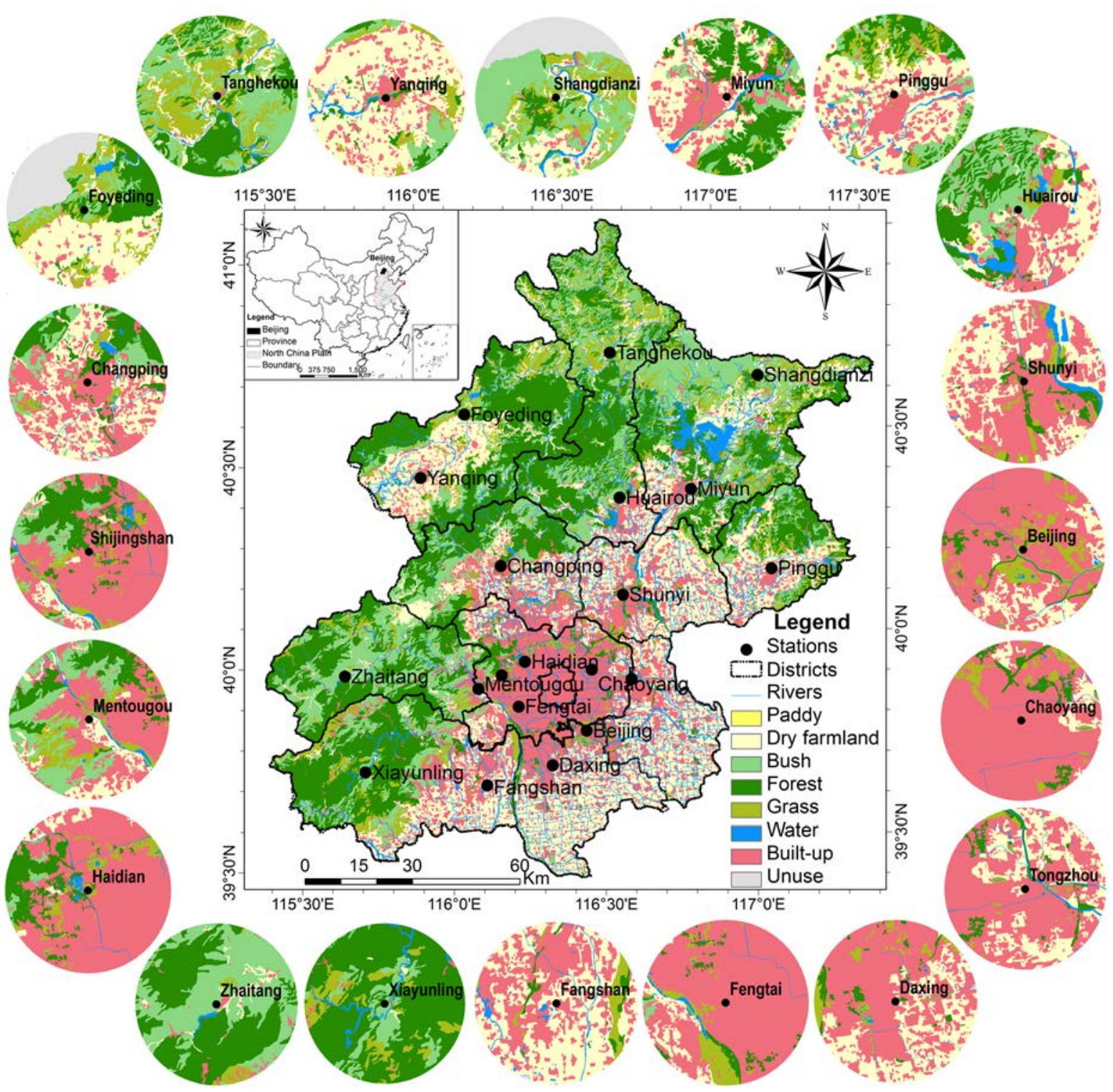

Figure 1. Locations of study area, 20 weather stations and land use in $10 \mathrm{~km}$ buffer. 


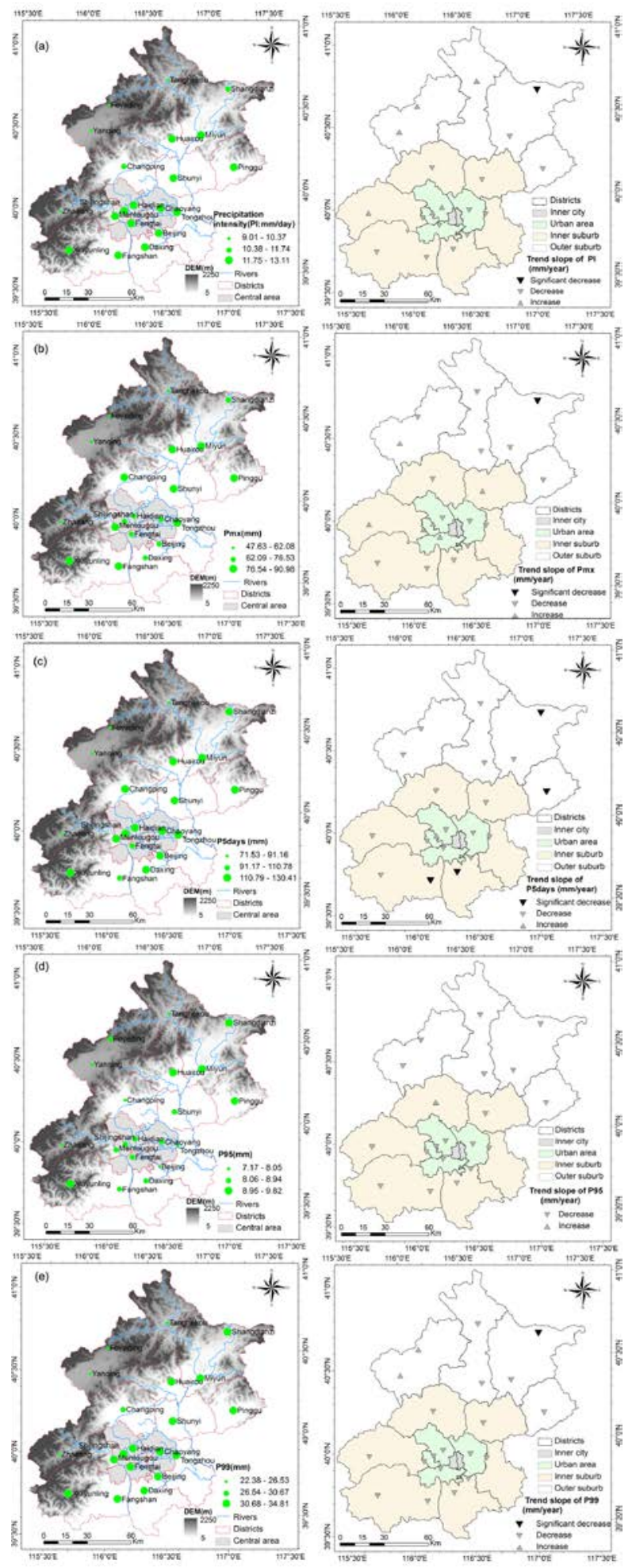


Figure 2. Spatial and temporal variations of magnitude metrics of extreme precipitation (a: PI; b: Pmx; c: P5days; d: P95; e: P99). 

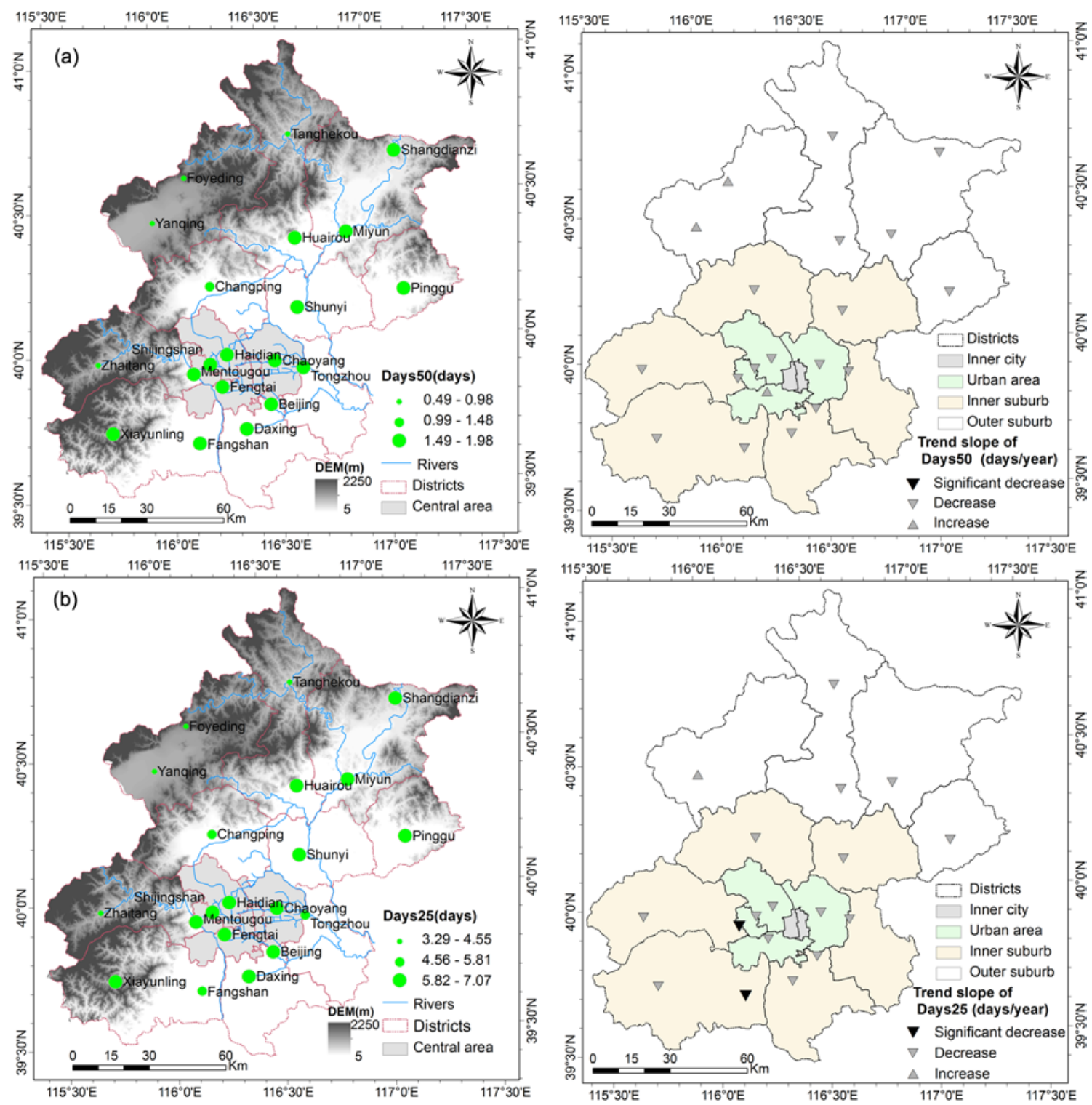

Figure 3. Spatial and temporal variations of frequency metrics of extreme precipitation (a: Days50; b: Days25). 

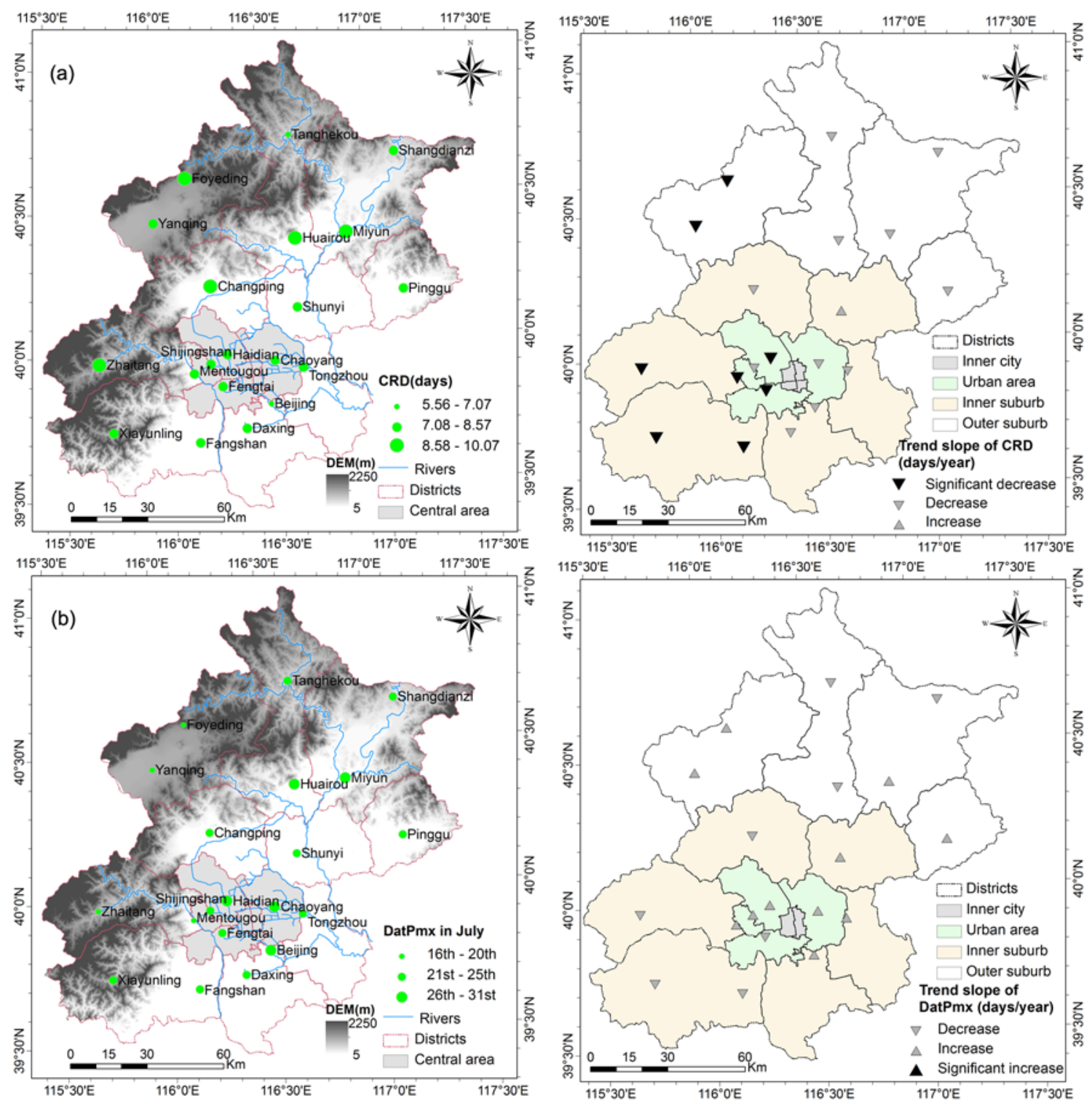

Figure 4. Spatial and temporal variations of duration (a: CRD) and timing (b: DatPmx) metrics of extreme precipitation. 

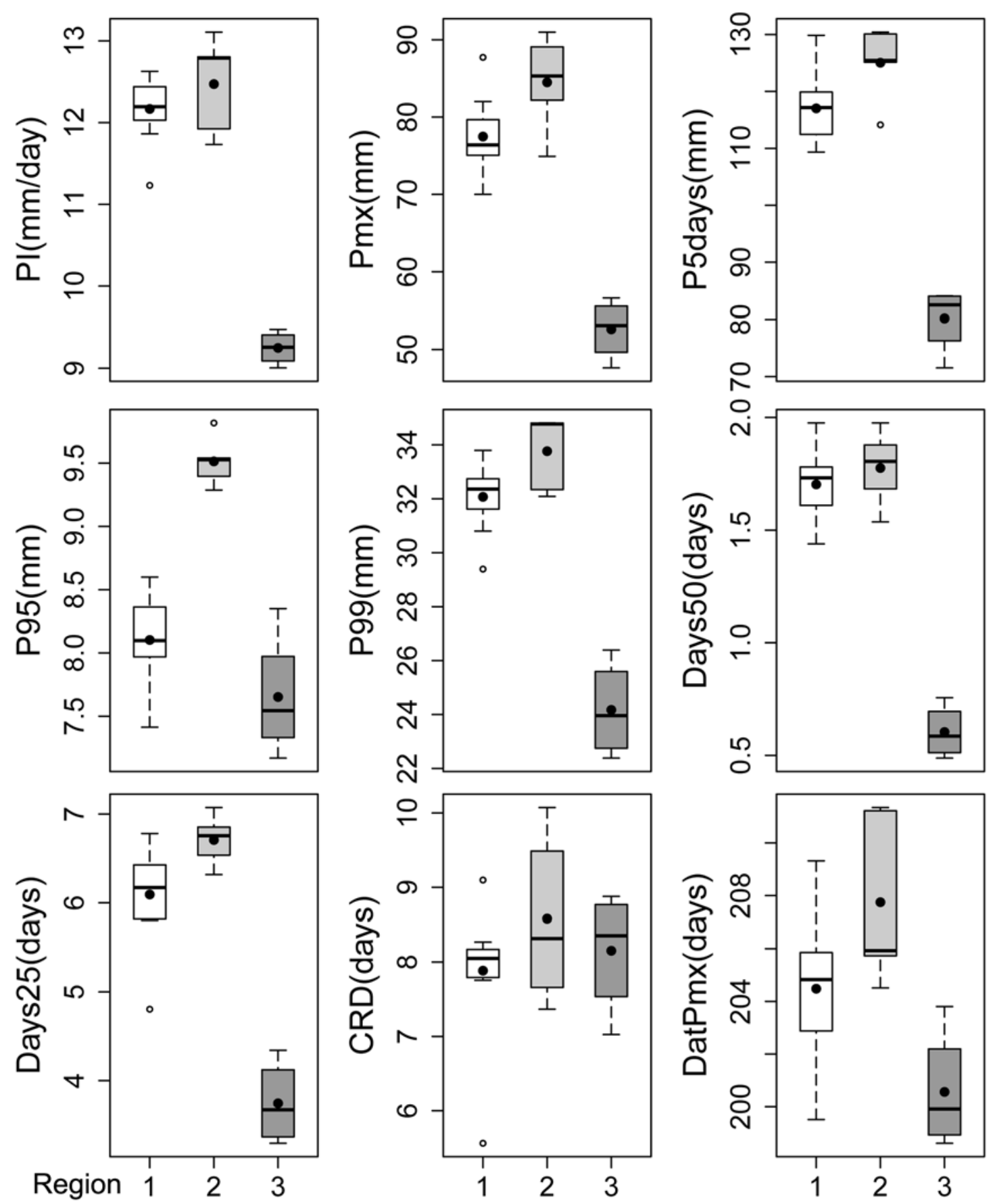
Figure 5. Metric discrepancies of extreme precipitation among different regions. Boxes define the 25th, median and 75th percentile values, and the vertical bars (whiskers) define the 10th and 90th percentile values. Mean values are defined by the solid dot symbols, respectively. 


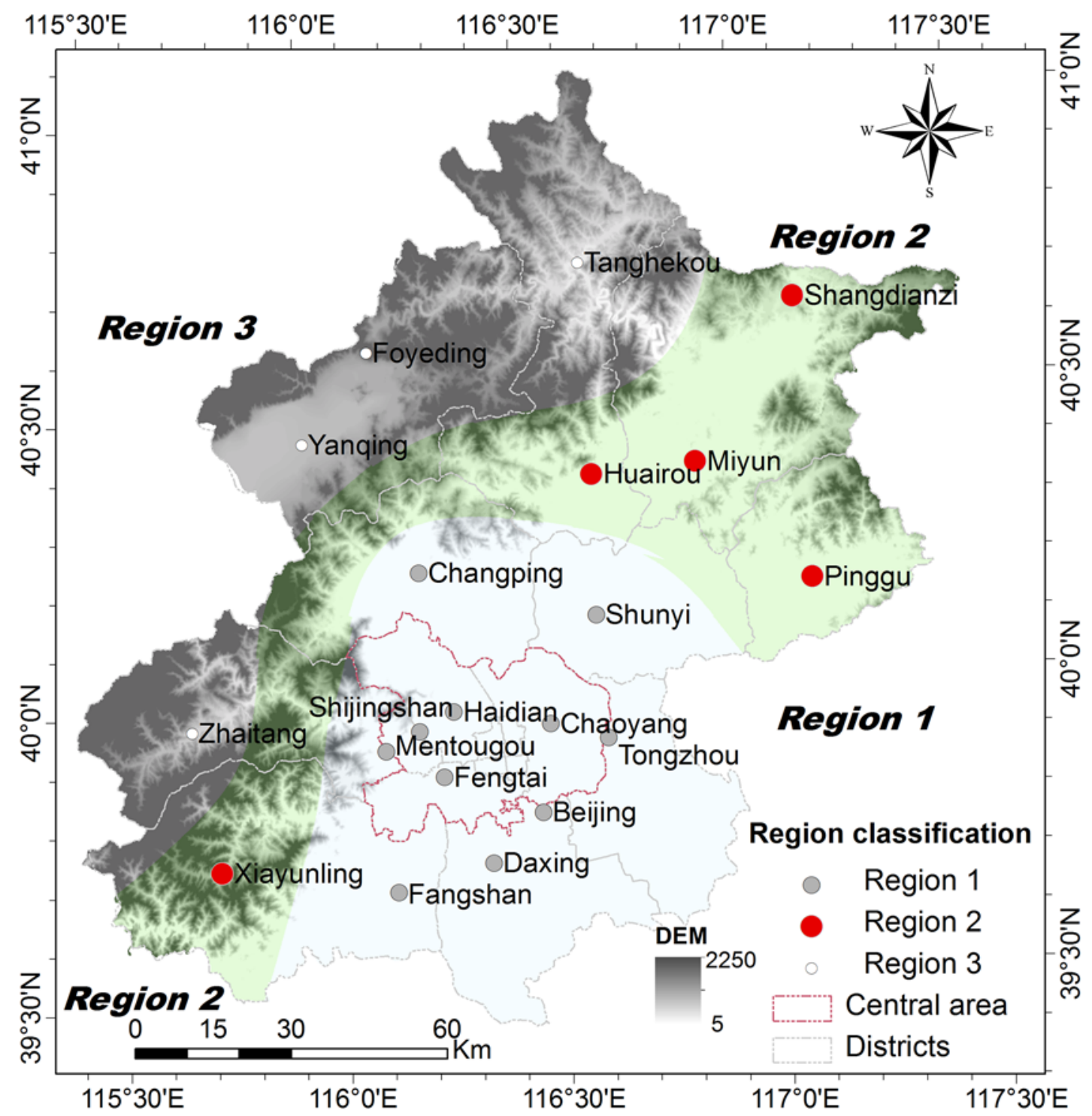

Figure 6. Regional classification of extreme precipitation at 20 weather stations by cluster analysis. Region 1 is the central urban region, Region 2 is the windward slope of topographic area and Region 3 is the mountainous area in the outer suburb. 

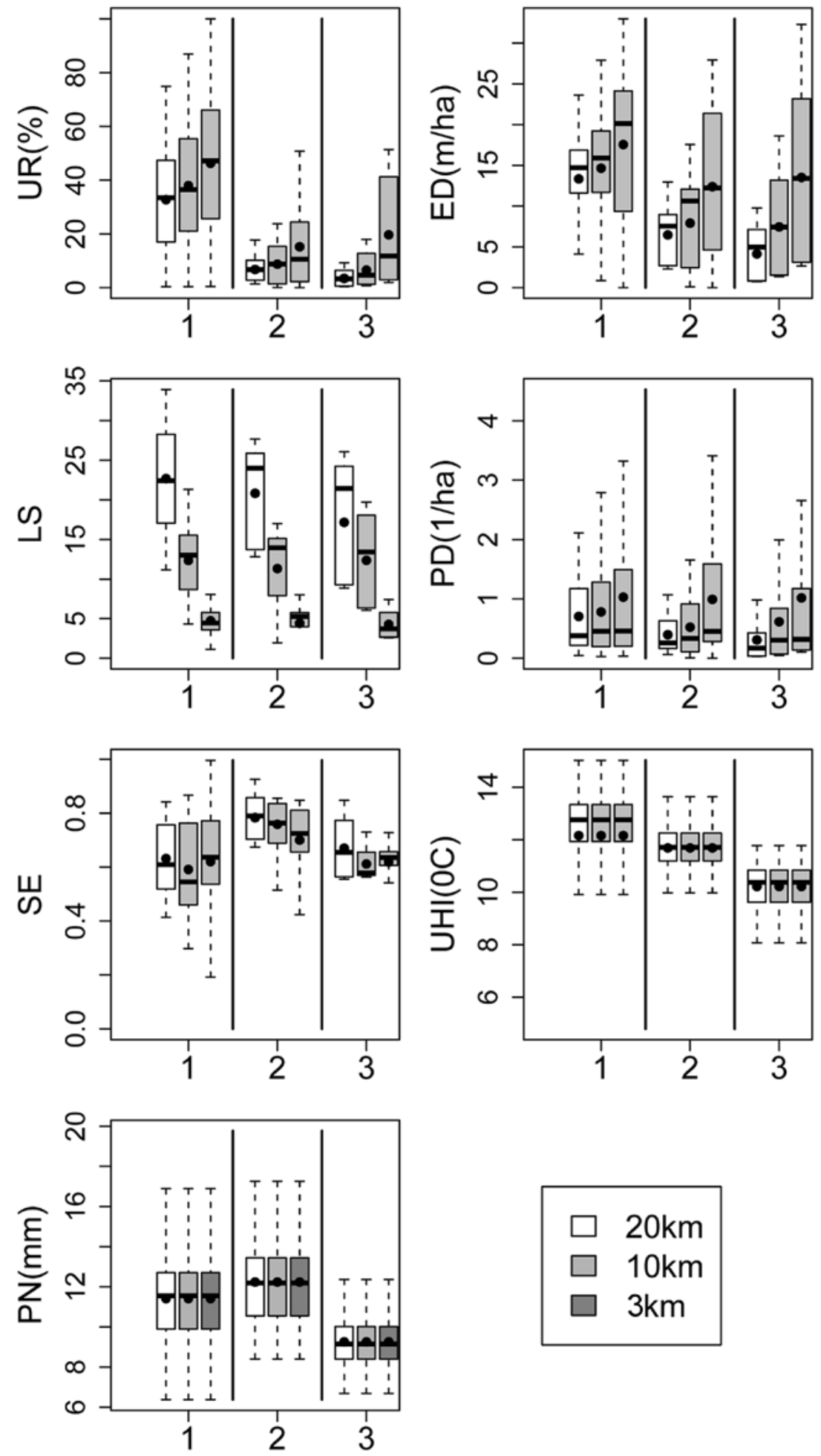
Figure 7. Regional discrepancies of urbanization metrics. Boxes define the 25th, median and 75th percentile values, and the vertical bars (whiskers) define the 10th and 90th percentile values. Mean values are defined by the solid dot symbols, respectively. 


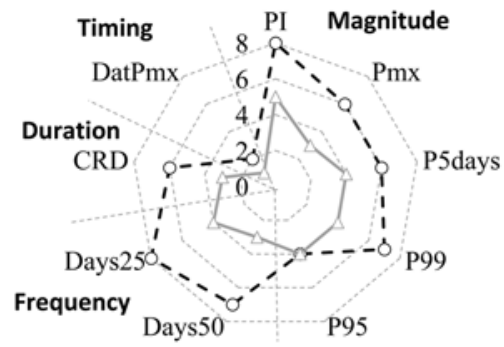

(a) $3 \mathrm{~km}$ buffer

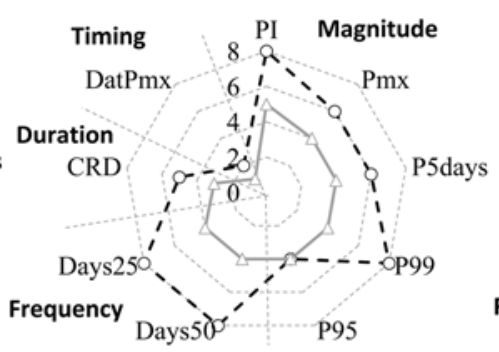

(b) $10 \mathrm{~km}$ buffer

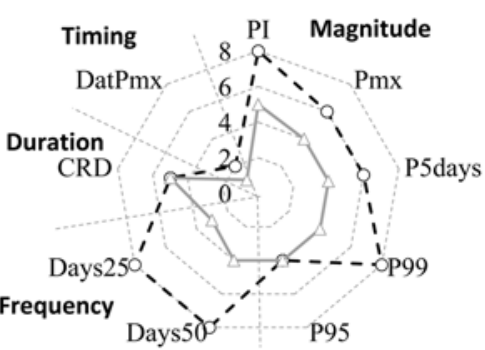

(c) $20 \mathrm{~km}$ buffer

$$
-\infty \text { Region } 1 \quad \longrightarrow \text { Region2 }
$$

Figure 8. Numbers of extreme precipitation metrics in the highly urbanized regions (Regions 1 and 2) which are statistically significant in the $95 \%$ confidence interval by Granger causal relationship detection for $3 \mathrm{~km}$ (a), $10 \mathrm{~km}$ (b) and $20 \mathrm{~km}$ (c) concentric buffers. 


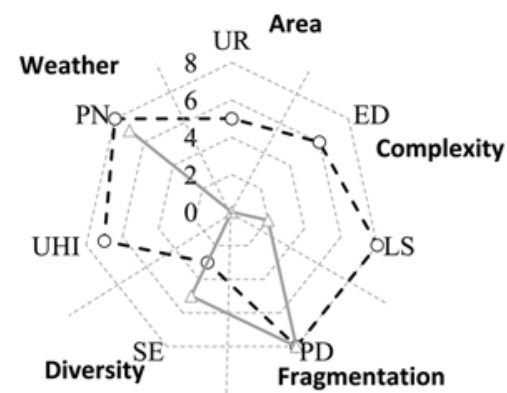

(a) $3 \mathrm{~km}$ buffer

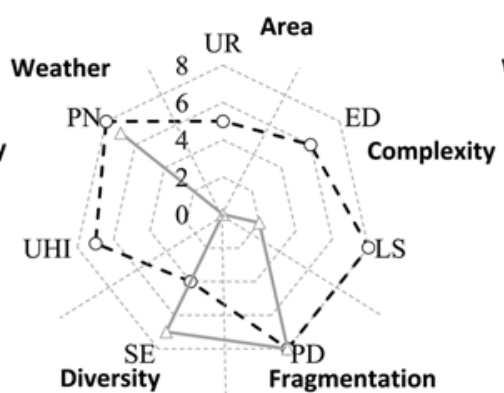

(b) $10 \mathrm{~km}$ buffer

$$
\text { - - Region1 } \rightarrow-\text { Region2 }
$$

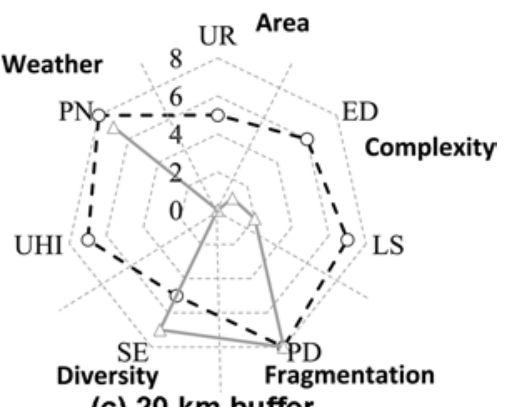

(c) $20 \mathrm{~km}$ buffer

Figure 9. Numbers of urbanization metrics in the highly urbanized regions (Regions 1 and 2) which are statistically significant in the $95 \%$ confidence interval by Granger causal relationship detection for $3 \mathrm{~km}$ (a), $10 \mathrm{~km}$ (b) and $20 \mathrm{~km}$ (c) concentric buffers. 
2015 lucc

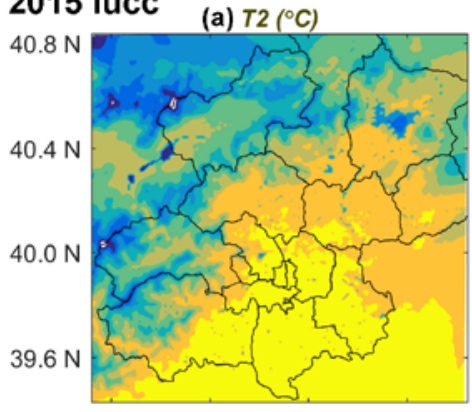

115.5E 116.0 E 116.5 E 117.0E

1980s lucc

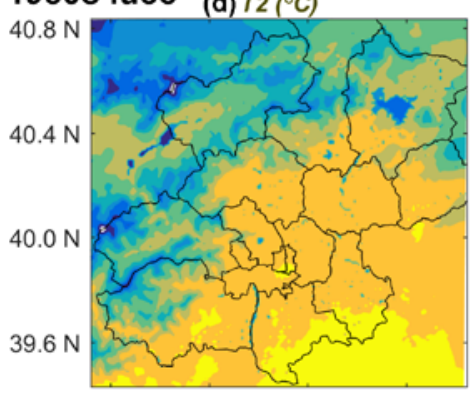

115.5 E 116.0 E 116.5 E 117.0 E

Difference $(\mathrm{g}) \Delta \mathrm{T} 2\left({ }^{\circ} \mathrm{C}\right)$

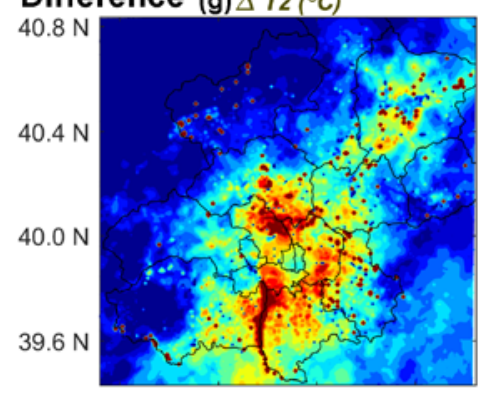

115.5 E 116.0 E 116.5 E 117.0 E (b) PSFC (Pa)

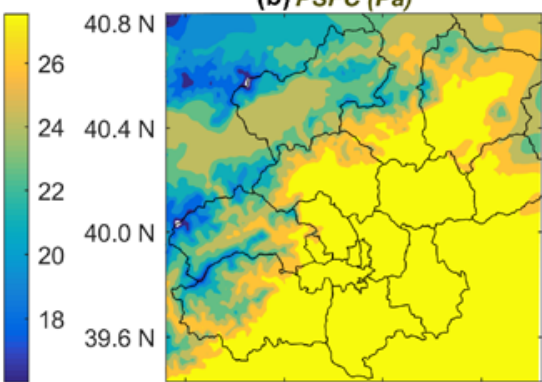

115.5 E 116.0 E 116.5 E 117.0 E

(e) PSFC ( $\mathrm{Pa})$

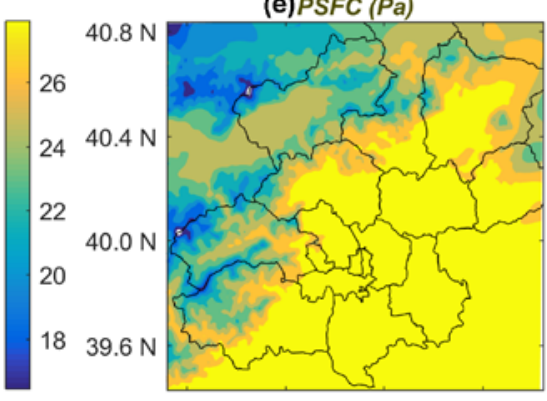

115.5 E 116.0 E 116.5 E 117.0 E

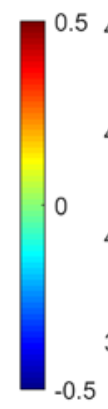

(h) $\triangle P S F C(\mathrm{~Pa})$

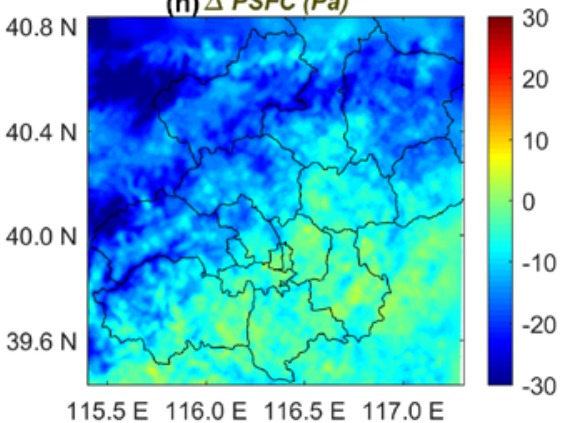

(c) Wind $(\mathrm{m} / \mathrm{s})$

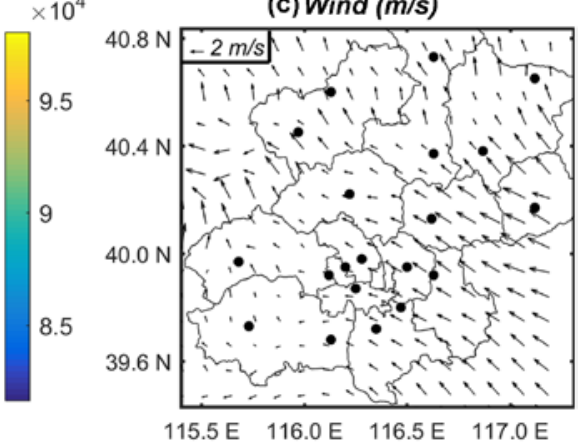

$\times 10^{4}$
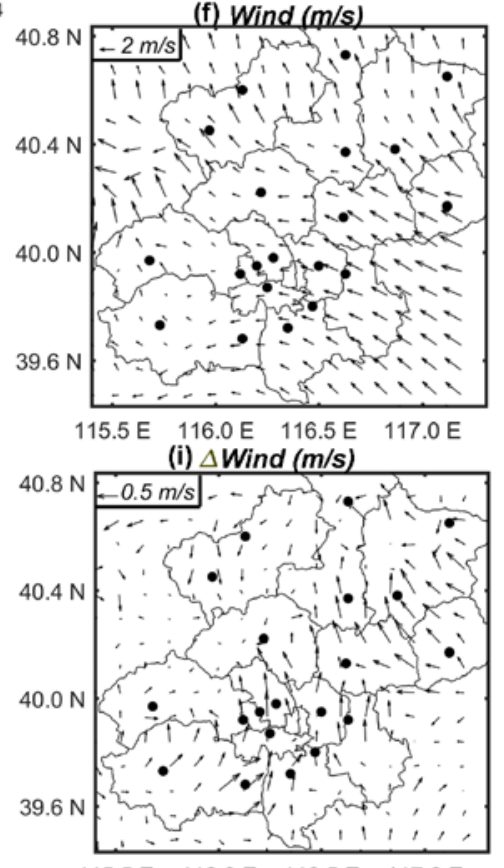

Figure 10. Simulated fields of air pressure (PSFC, $\left.10^{4} \mathrm{~Pa}\right)$, air temperature $\left(\mathrm{T},{ }^{\circ} \mathrm{C}\right)$ and wind $(\mathrm{m} / \mathrm{s})$ during torrential precipitation under the 1980s (d, e and f) and 2015 (a, b and c) land use scenarios, as well as their regional differences ( $\mathrm{g}, \mathrm{h}$ and $\mathrm{i}$ ). 


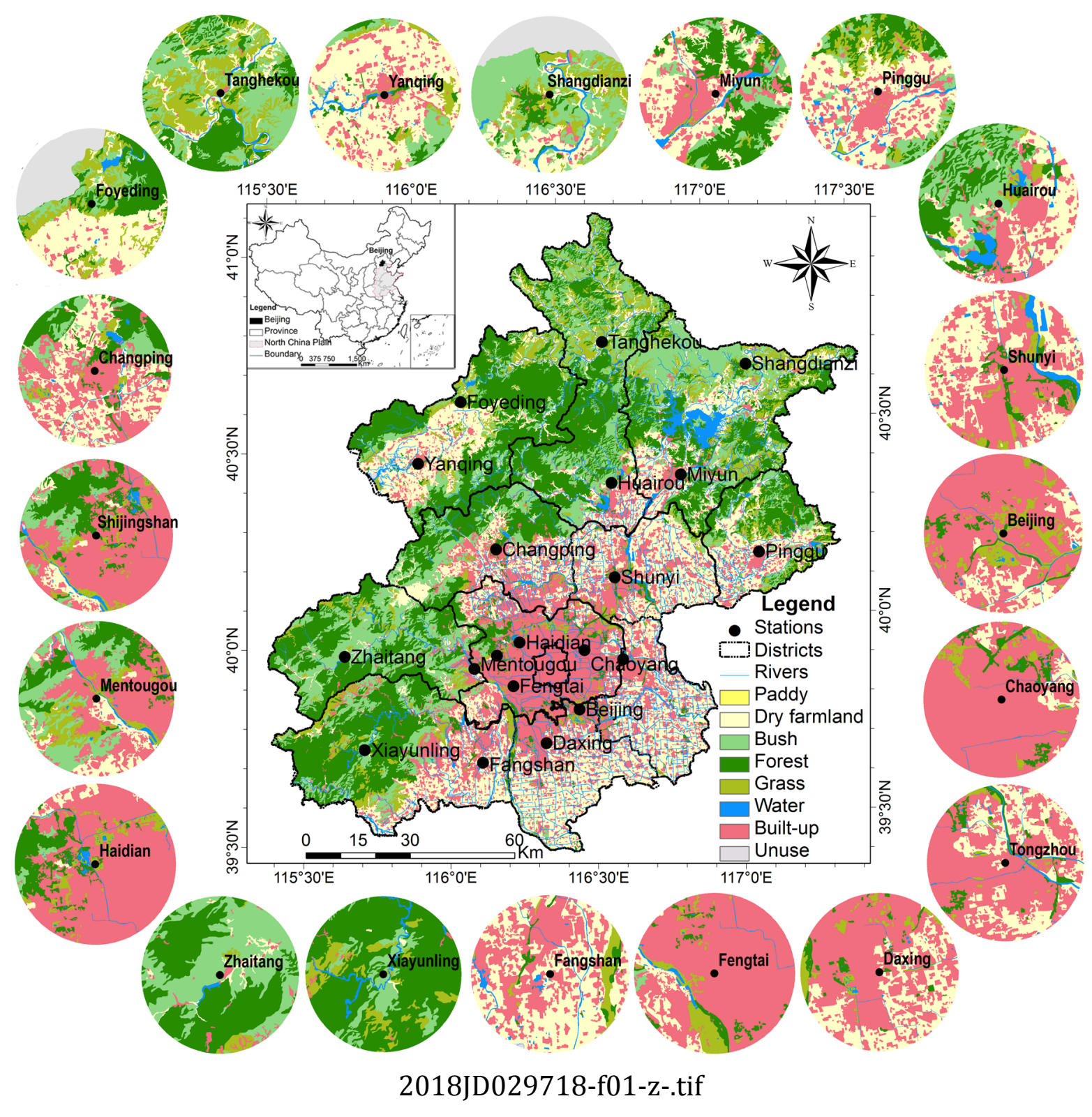

This article is protected by copyright. All rights reserved. 


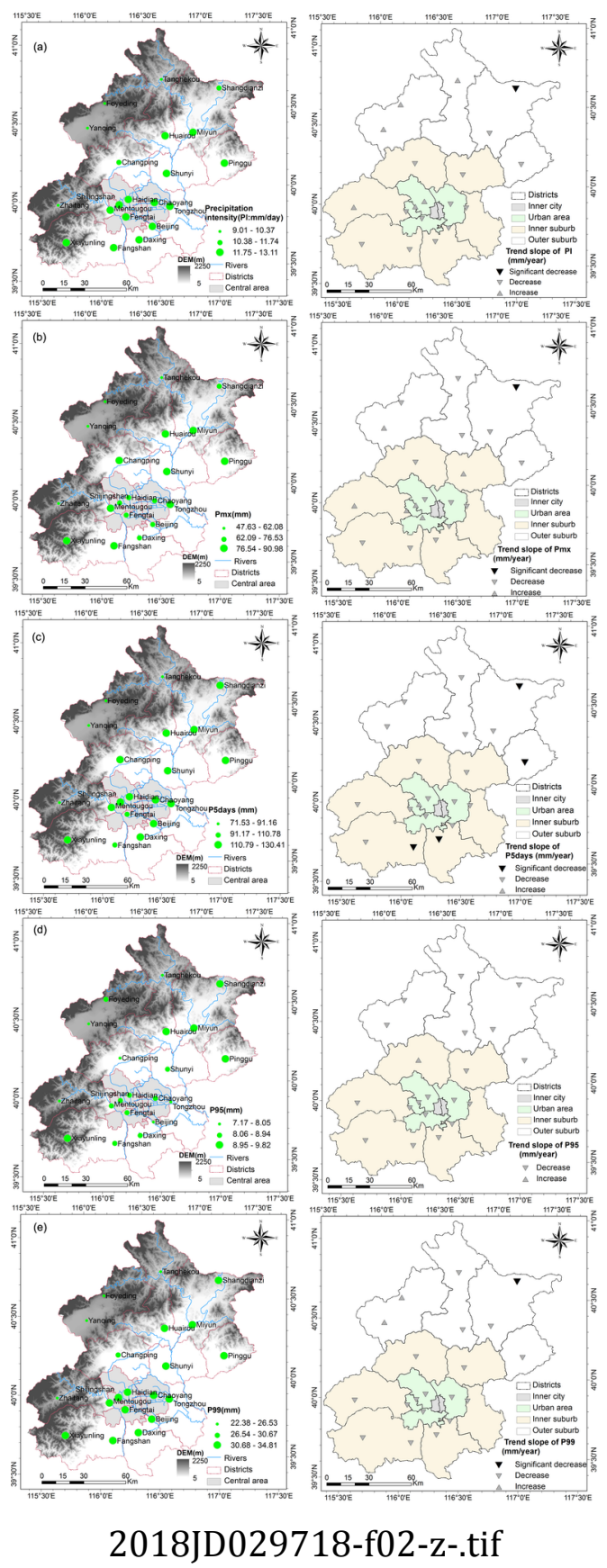

This article is protected by copyright. All rights reserved. 

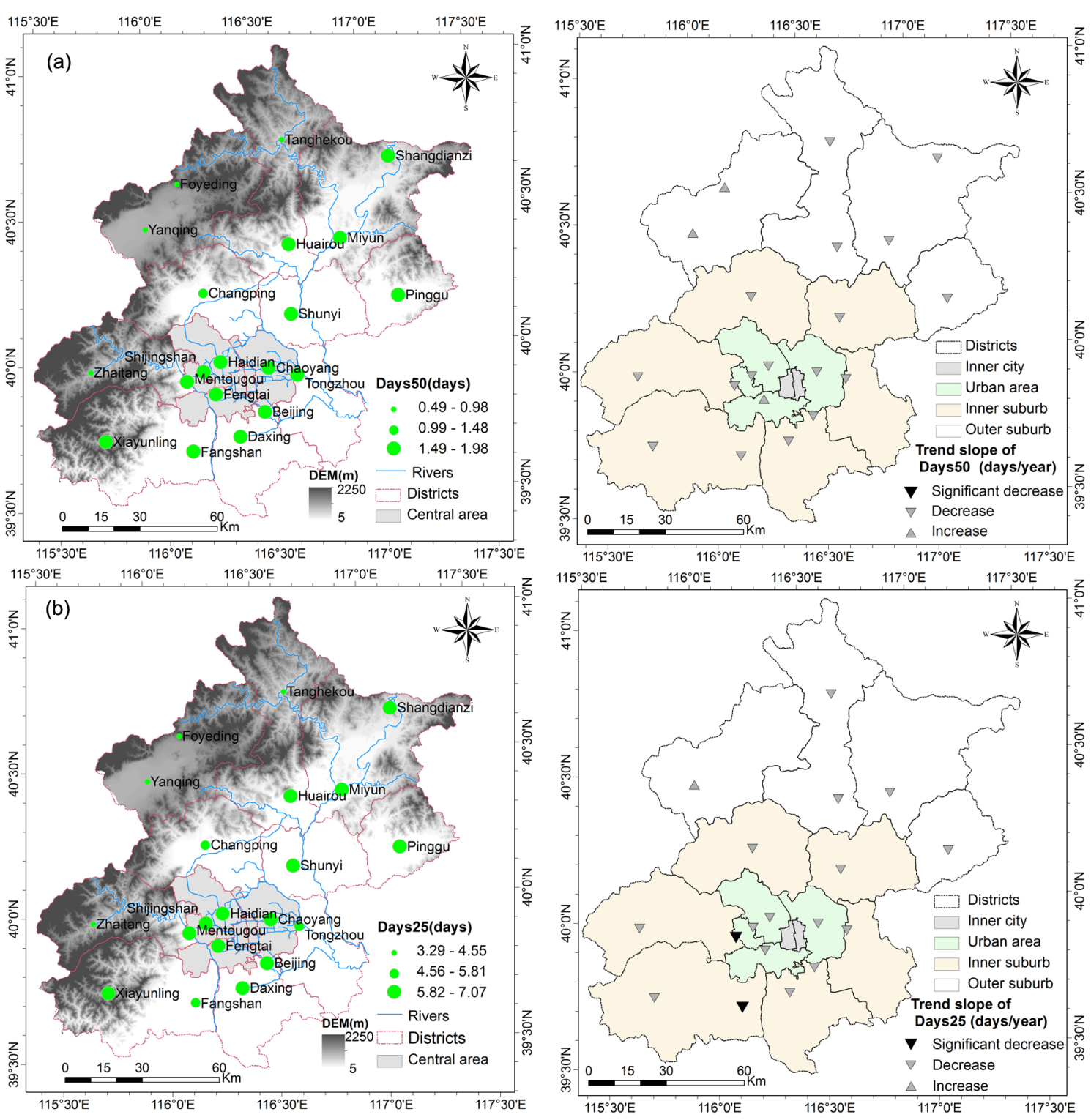

2018JD029718-f03-z-.tif 

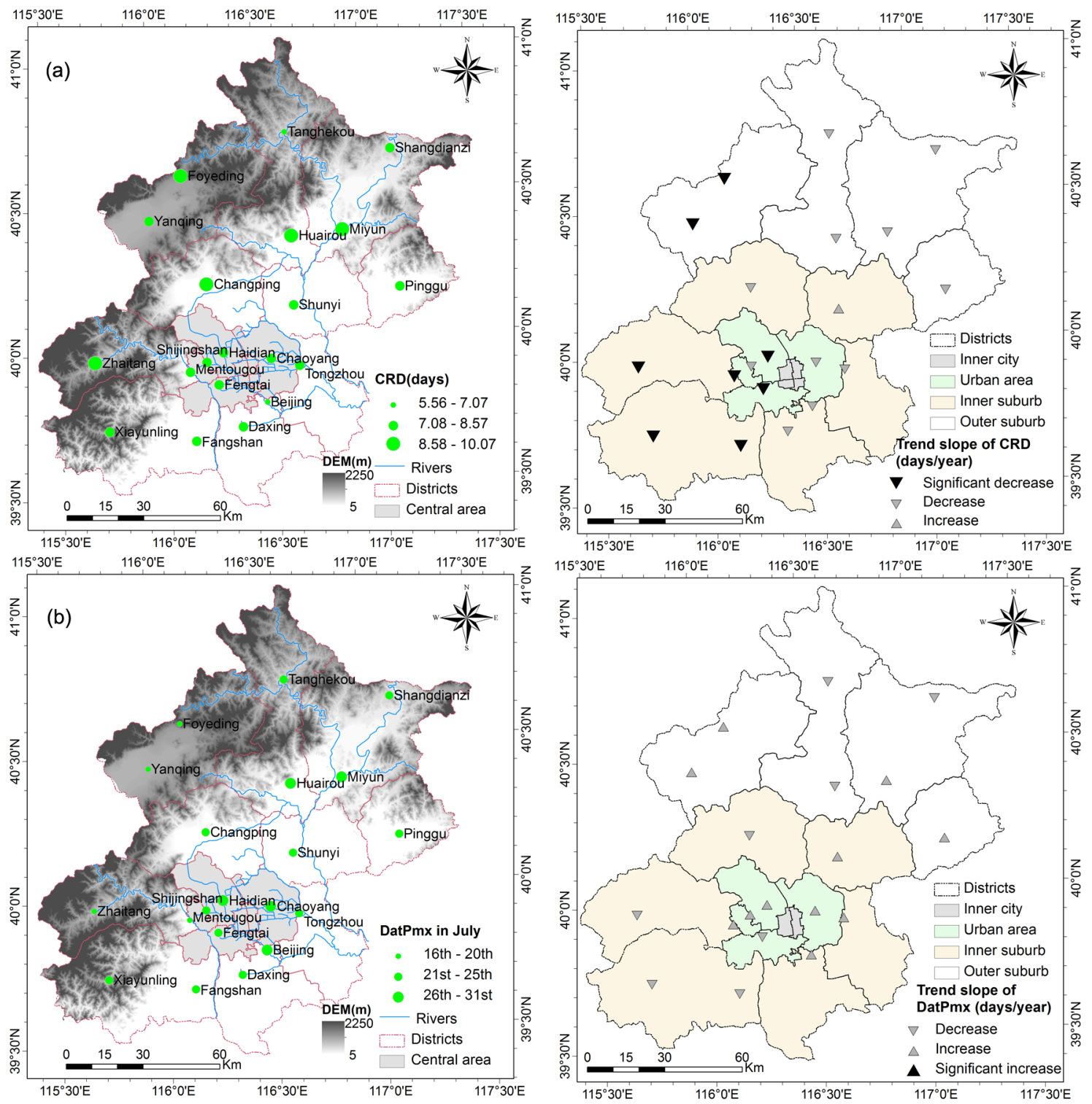

2018JD029718-f04-z-.tif 

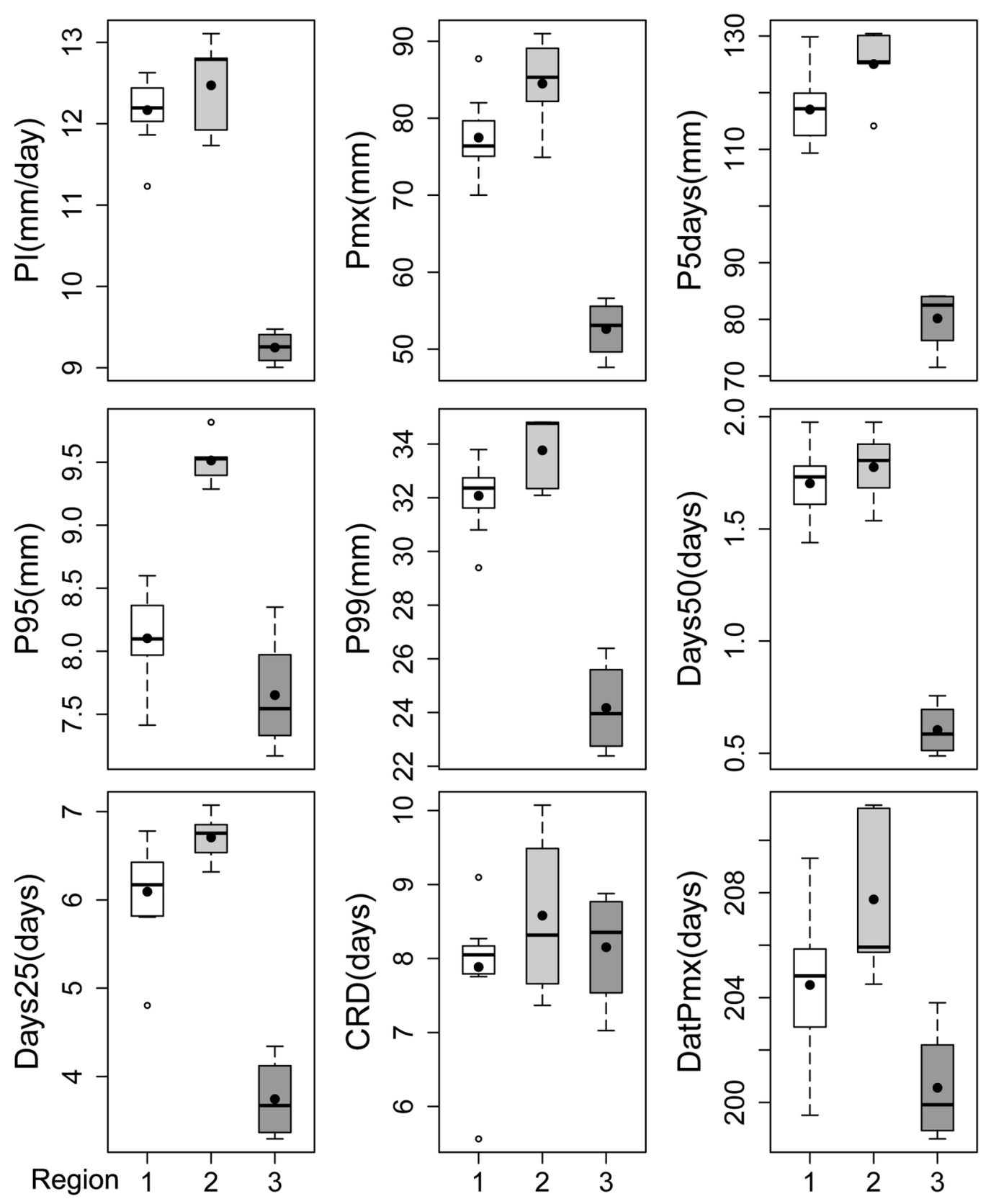

2018JD029718-f05-z-.tif 


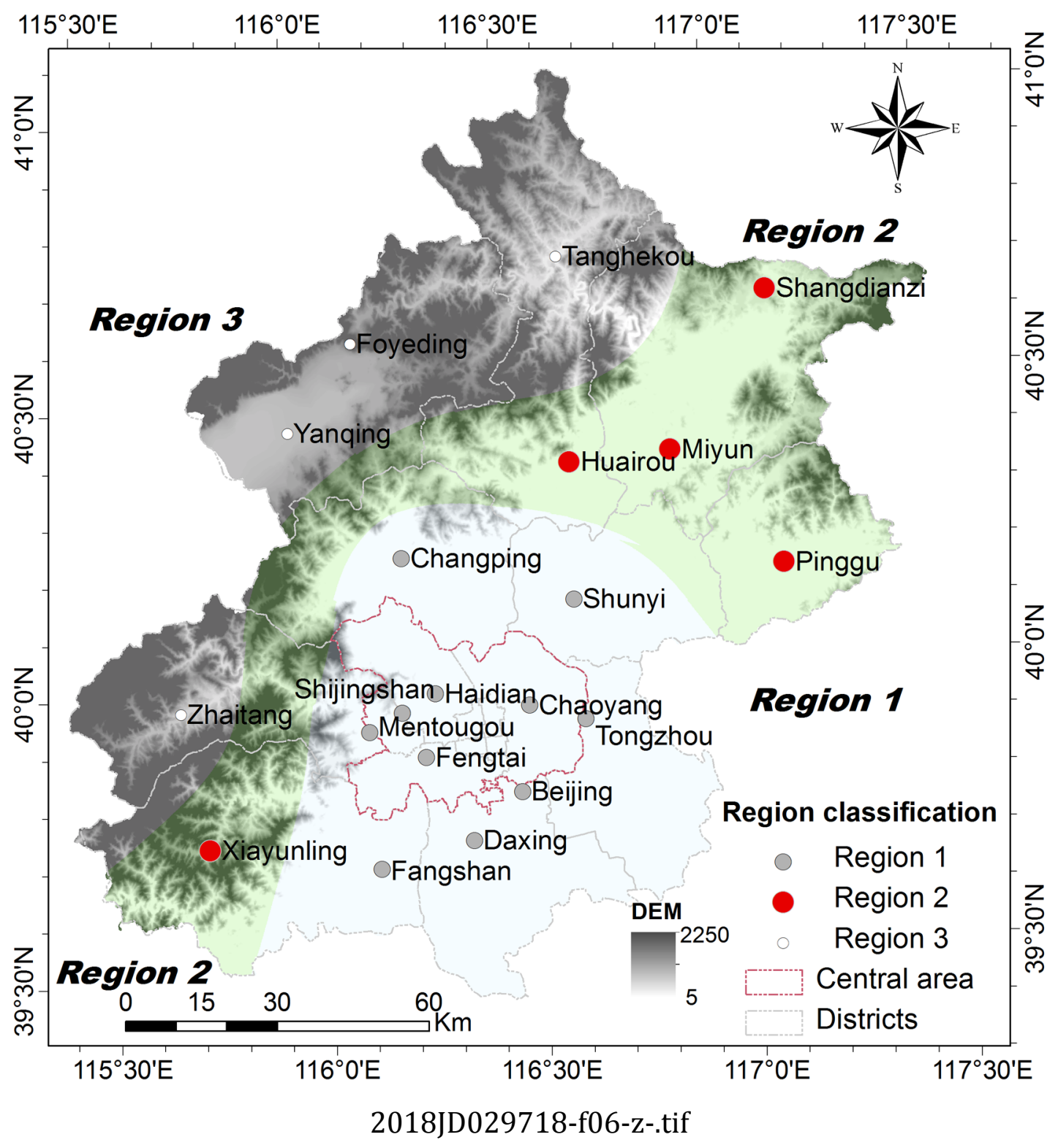

This article is protected by copyright. All rights reserved. 

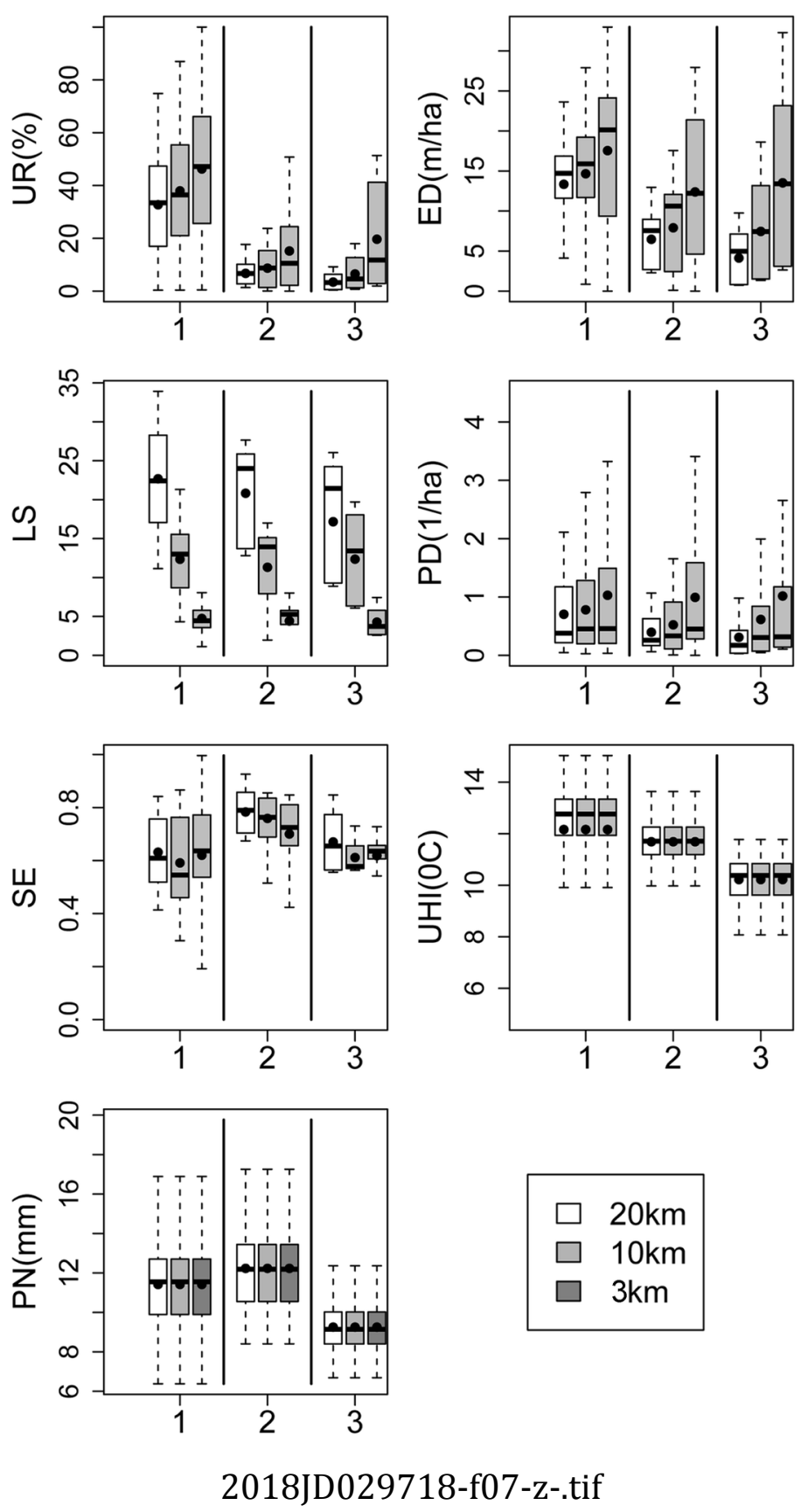

This article is protected by copyright. All rights reserved. 


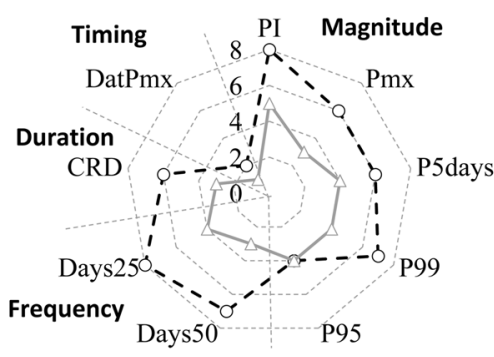

(a) $3 \mathrm{~km}$ buffer

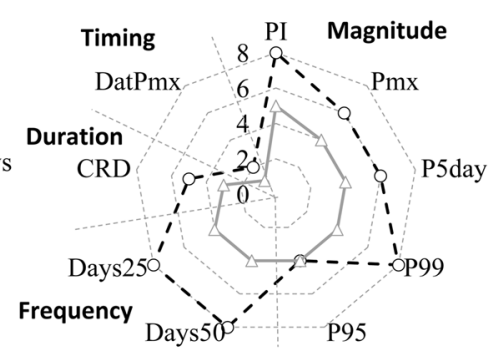

(b) $10 \mathrm{~km}$ buffer

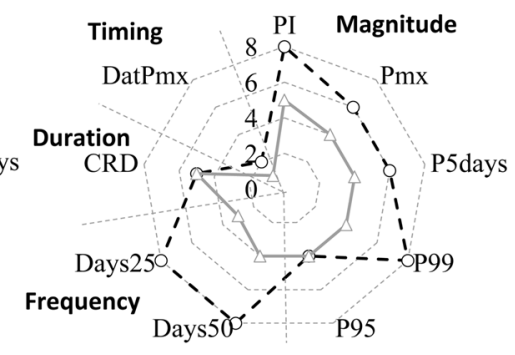

(c) $20 \mathrm{~km}$ buffer - - Region1 $\quad--$ Region2 2018JD029718-f08-z-.tif 


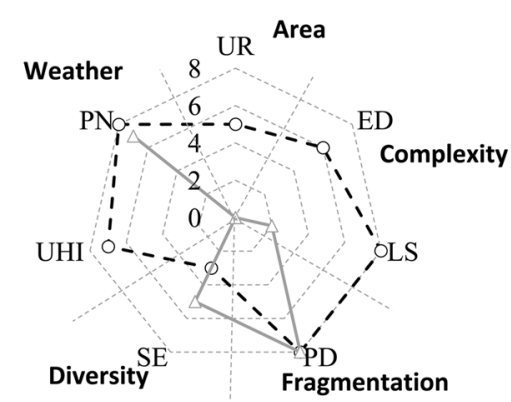

(a) $3 \mathrm{~km}$ buffer

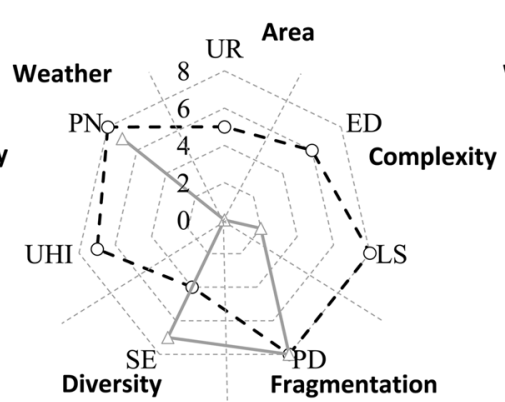

(b) $10 \mathrm{~km}$ buffer

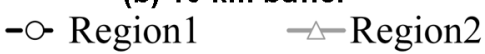
2018JD029718-f09-z-.tif

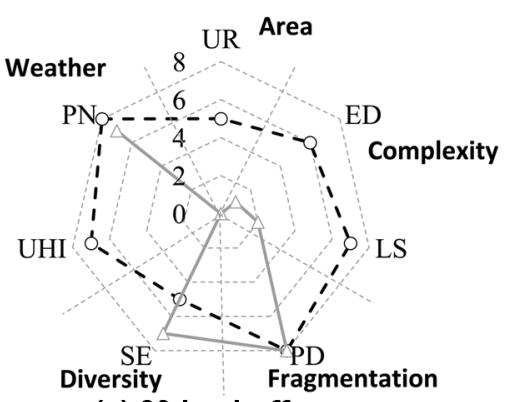

(c) $20 \mathrm{~km}$ buffer 
2015 lucc

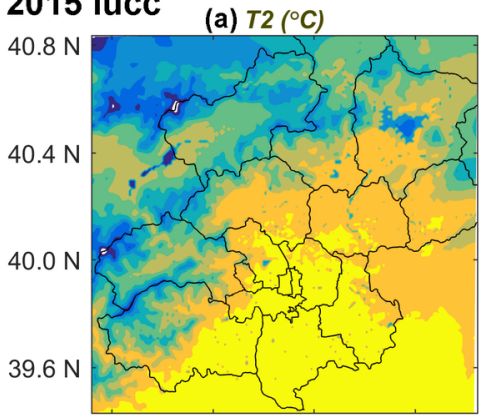

115.5 E 116.0 E 116.5 E 117.0 E

\section{0s lucc}

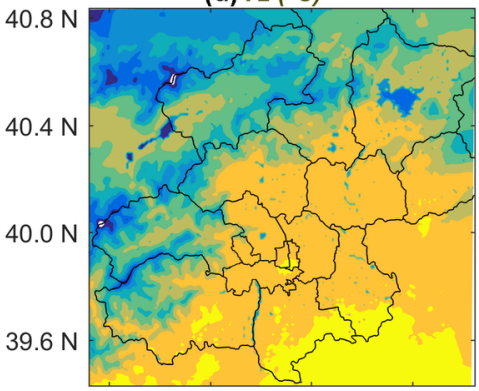

115.5 E 116.0 E 116.5E 117.0 E Difference $(\mathrm{g}) \Delta T 2\left({ }^{\circ} \mathrm{C}\right)$

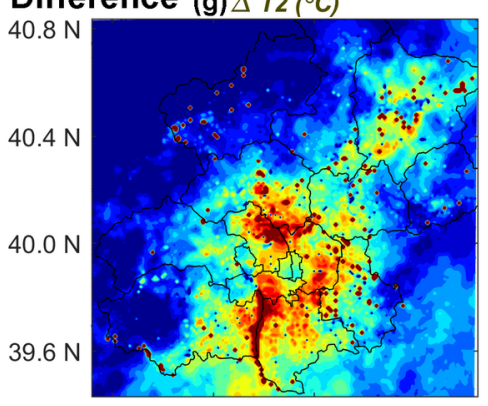

115.5 E 116.0 E 116.5 E 117.0 E (b) PSFC ( $\mathrm{Pa})$

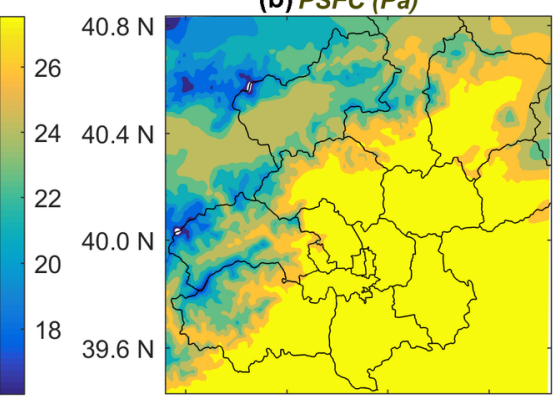

115.5 E 116.0 E 116.5 E 117.0 E

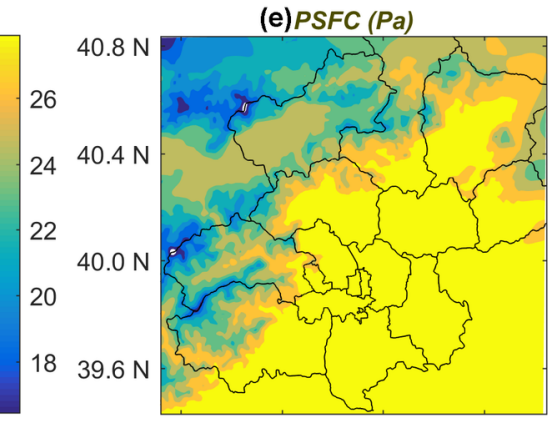

115.5 E 116.0 E 116.5 E 117.0 E (h) $\triangle$ PSFC (Pa)
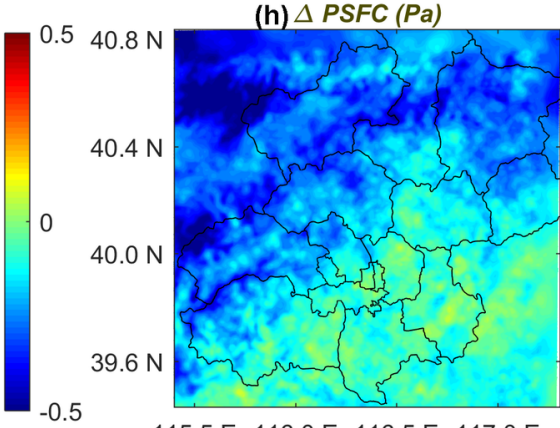

115.5 E 116.0 E 116.5 E 117.0 E 2018JD029718-f10-z-.tif $\times 10^{4}$

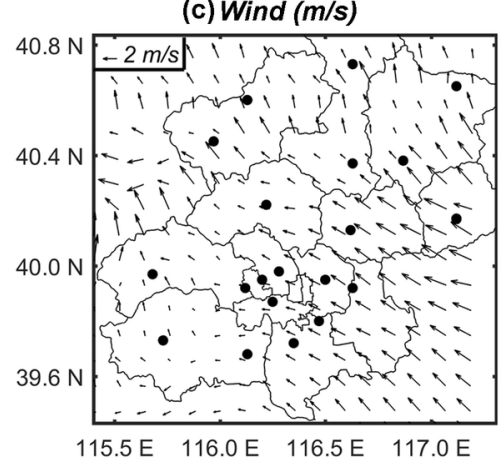

$\times 10^{4}$ (f) Wind (m/s)
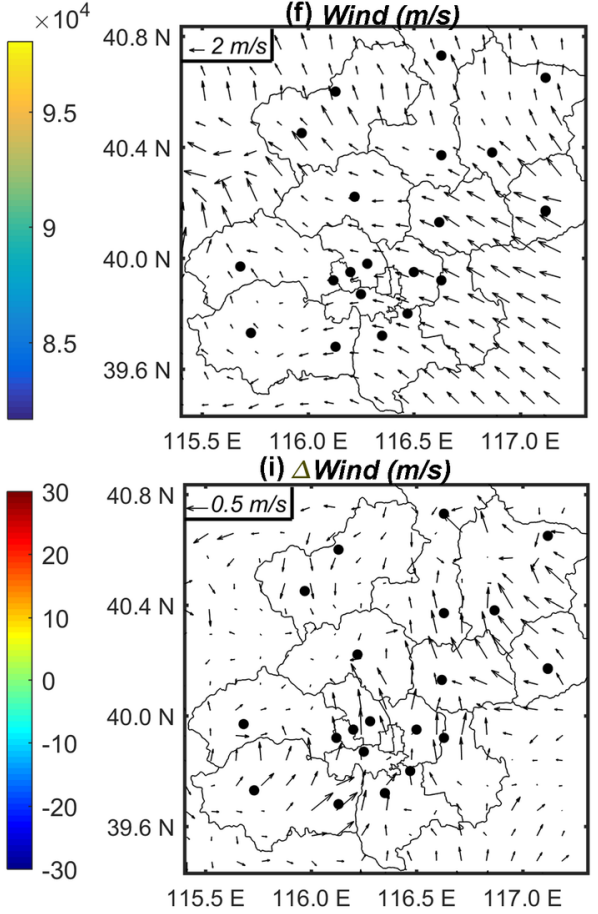